\title{
Disc-mass distribution in star-disc encounters ${ }^{\star}$
}

\author{
M. Steinhausen ${ }^{1,2}$, C. Olczak ${ }^{3,4,5}$, and S. Pfalzner ${ }^{1}$ \\ 1 Max-Planck-Institut für Radioastronomie (MPIfR), Auf dem Hügel 69, 53121 Bonn, Germany \\ e-mail: mstein@mpifr-bonn.mpg.de \\ 2 I. Physikalisches Institut, Universität zu Köln, Zülpicher Str. 77, 50937 Köln, Germany \\ 3 Astronomisches Rechen-Institut (ARI), Zentrum für Astronomie der Universität Heidelberg (ZAH) Mönchhofstr. 12-14, \\ 69120 Heidelberg, Germany \\ 4 Max-Planck-Institut für Astronomie (MPIA), Königstuhl 17, 69117 Heidelberg, Germany \\ 5 National Astronomical Observatories of China (NAOC), Chinese Academy of Sciences (CAS), 20A Datun Lu, Chaoyang District, \\ Beijing 100012, PR China
}

Received 11 July 2011 / Accepted 10 November 2011

\section{ABSTRACT}

\begin{abstract}
Aims. Investigations of stellar encounters in cluster environments have demonstrated their potential influence on the mass and angular momentum of protoplanetary discs around young stars. We investigated how far the initial surface density in the disc surrounding a young star influences the outcome of an encounter.

Methods. The numerical method applied here allows us to determine the mass and angular momentum losses in an encounter for any initial disc-mass distribution. On the basis of a power-law ansatz for the surface density, $\Sigma(r) \propto r^{-p}$, we perform a parameter study of star-disc encounters with different initial disc-mass distributions using N-body simulations.

Results. We demonstrate that the shape of the disc-mass distribution has a significant impact on the quantity of the disc-mass and angular momentum losses in star-disc encounters. In particular, the results are most sensitive to how the outer parts of the disc are perturbed by high-mass stars. In contrast, disc-penetrating encounters lead more or less independently of the disc-mass distribution always to large losses. However, maximum losses are generally obtained for initially flat distributed disc material. Based on a parameter study, a fit formula is derived, describing how the relative mass and angular momentum loss depend on the initial disc-mass distribution index $p$. Encounters generally lead to a steepening of the density profile of the disc. The resulting profiles can have a $r^{-2}$-dependence or an even steeper one that is independent of the initial distribution of the disc material.

Conclusions. From observations, the initial density distribution in discs remains unconstrained, hence the strong dependence on the initial density distribution that we find here might require a revision of the effect of encounters in young stellar clusters. The steep surface density distributions induced by some encounters might be a prerequisite to the formation of planetary systems similar to our own Solar System.
\end{abstract}

Key words. methods: numerical - protoplanetary disks - circumstellar matter

\section{Introduction}

There is increasing observational evidence that most, if not all, stars are initially surrounded by a circumstellar disc. For example, Lada et al. (2000) found by examining the $L$-band excess of young stars in the Trapezium cluster that a fraction of $80-85 \%$ of all stars are surrounded by discs. With time, these protoplanetary discs become depleted of gas and dust and eventually disappear (Haisch et al. 2001; Hillenbrand 2002; Sicilia-Aguilar et al. 2006; Hernández et al. 2007; Currie et al. 2008; Hernández et al. 2008; Mamajek 2009; Massi et al. 2010). It is currently unclear which of a variety of physical mechanisms dominates this evolutionary processes, among them internal processes such as viscous torques (e.g. Shu et al. 1987), turbulent effects (Klahr \& Bodenheimer 2003), and magnetic fields (Balbus \& Hawley 2002), while photoevaporation (Scally \& Clarke 2001; Clarke et al. 2001; Matsuyama et al. 2003; Johnstone et al. 2004; Alexander et al. 2005, 2006; Ercolano et al. 2008; Drake et al. 2009; Gorti \& Hollenbach 2009) and tidal torques (Heller 1993; Clarke \& Pringle 1993; Ostriker 1994; Heller 1995; Hall et al. 1996; Hall 1997; Larwood 1997; Boffin et al. 1998; Pfalzner 2004; Pfalzner et al. 2005a; Moeckel \& Bally 2006; Kley et al. 2008) are candidates for external disc destruction processes.

\footnotetext{
* Appendices A and B are available in electronic form at http://www. aanda.org
}

The focus of the present numerical investigation is the effect of gravitational star-disc interactions on the disc-mass distribution and, therefore, the mass and angular momentum of the discs. A star-disc encounter ${ }^{1}$ can cause matter to become unbound, be captured by the perturbing star or pushed inwards and potentially be accreted by the central star. The extent to which this happens depends on the periastron distance, the mass ratio of the stars, the eccentricity and, moreover, the initial (pre-encounter) mass distribution in the disc. To date, limitations in observations of protoplanetary discs have prevented the discovery of how consistently the surface density of low-mass discs develops. This means that owing to observational constraints a unique predetermined initial state for the disc-mass distribution before an encounter does not exist. A wide variety of surface densities have been derived by fitting resolved millimetre continuum or line emission data with parametric disc structure models (e.g. Mundy et al. 1996; Lay et al. 1997) or in combination with broadband spectral energy distributions (SEDs) (Wilner et al. 2000; Testi et al. 2001; Akeson et al. 2002; Kitamura et al. 2002; Andrews $\&$ Williams 2007b). While those studies have profoundly shaped our knowledge of disc structures, they have all fundamentally been limited by the low angular resolution of available data.

\footnotetext{
1 The term "star-disc encounter" means here encounters in which only one of the stars is surrounded by a disc, in contrast to disc-disc encounters, which are encounters in which both stars are surrounded by discs.
} 
The standard fitting method underlies the assumption that the surface density $\Sigma$ has a simple power-law dependence of the form

$\Sigma(r) \propto r^{-p}$

out to some cut-off radius (e.g. Andrews \& Williams 2007a). Estimates based on numerical power-law models fitted to observational data lead to distribution indices $p$ ranging roughly from $p=0$ to $p=2$. Recent studies have even found anomalous results, e.g. Isella et al. (2009) who measured distribution indices of $p<0$.

Analytical approaches have also proposed different discmass distribution indices. The most widely used model is that of a steady-state viscous accretion disc with a surface density distribution index of $p=1$ (e.g. Hartmann et al. 1998). However, simulations of the evolution of protostellar discs that form selfconsistently from the collapse of a molecular cloud core have uncovered a surface distribution index of $p=1.5$ (Lin \& Pringle 1990; Hueso \& Guillot 2005; Vorobyov \& Basu 2007), while studies that include magnetised disc material have found a flatter disc-mass distribution of $p=0.75$ (Shu et al. 2007). Taking this huge variety of distributions into account, one has to consider different initial disc-mass distributions to evaluate their effect in star-disc encounters.

Most previous numerical studies of star-disc encounters have used only a single density distribution, mainly focusing on the case of a theoretically motivated $r^{-1}$ disc-mass distribution (Hall et al. 1996; Hall 1997; Pfalzner 2004; Olczak et al. 2006; Moeckel \& Bally 2006; Pfalzner \& Olczak 2007). Star-disc encounters with different initial disc-mass distributions have only been considered in a very limited way. Heller (1995) performed numerical simulations of two different mass distributions ( $p=0$ and $p=1.5$ ) concentrating on parabolic encounters with equal mass stars, while Hall (1997) investigated initial surface distributions of $p=0$ and $p=1$ for close and penetrating encounters of unity mass ratio. A study of a wide parameter range focusing on multiple initial disc-mass distributions still needs to be performed.

Nevertheless, numerical studies of star-disc encounters only allow a sectional view of the processes since it is impossible to simulate each combination of encounter parameters. Earlier analytical studies by Ostriker (1994) did not suffer from this shortcoming. In her study, a first order approximation of the angular momentum loss dependent on the initial disc-mass distribution is given. However, the validity of her results is limited to large periastron radii (for example $r_{\text {peri }} / r_{\text {disc }}>3$ for $M_{2} / M_{1}=1$ ), where the angular momentum loss is usually well below $10 \%$. Close or even penetrating encounters cannot be interpreted by this linear perturbation theory (Ostriker 1994; Pfalzner et al. 2005b) making numerical studies indispensable in this regime.

In this work, the effects of star-disc encounters are investigated for a large parameter space considering most configurations that can be expected in a typical young cluster. The investigated mass distributions cover the entire range of the so far observed disc-mass distributions.

Section 2 shortly describes the numerical methods and parameter range used in this study, while Sect. 3 presents the results of the simulations including a fit formula for the mass and angular momentum loss depending on the initial disc-mass distribution index $p$. A summary and discussion is given in Sect. 4.
Table 1. Initial conditions for all simulations.

\begin{tabular}{lc}
\hline \hline Variable & Value \\
\hline Outer disc radius, $r_{\text {disc }}$ & $100 \mathrm{AU}$ \\
Inner disc radius, $r_{\mathrm{i}, \text { disc }}$ & $10 \mathrm{AU}$ \\
Number of particles, $n_{\text {part }}$ & $10000(50000)$ \\
Disc mass, $m_{\text {disc }}$ & $10^{-4} M_{\odot}\left(10^{-3} M_{\odot}\right)$ \\
Particle distribution index, $b$ & $0,\left(\frac{7}{4}\right)$ \\
Mass distribution index, $p$ & $0, \frac{1}{2}, 1, \frac{7}{4}$ \\
\hline Rel. perturber mass, $M_{2} / M_{1}$ & $0.1-500$ \\
Rel. periastron distance, $r_{\text {peri }} / r_{\text {disc }}$ & $0.1-20$ \\
Eccentricity, $e$ & 1 \\
Simulation time, $t_{\text {end }}$ & $\sim 3000 \mathrm{yrs}$ \\
\hline
\end{tabular}

\section{Methods}

The encounter between a disc-surrounded star with a secondary star is modelled using a code that is based on the numerical method described in Pfalzner (2003). In our simulations, only one star is initially surrounded by a disc. However, previous investigations have shown that star-disc encounters can be generalised to disc-disc encounters as long as there is no significant mass exchange between the discs (Pfalzner et al. 2005a). In the case of a mass exchange, the discs can be to some extent replenished, such that for very close encounters the mass loss determined in this study would be slightly overestimated.

A summary of the disc properties can be found in Table 1. The disc is represented by 10000 pseudo-particles, distributed according to a given particle distribution $\propto r^{-b}$. Choosing this relatively small number of simulation particles is motivated by the aim to cover a large encounter parameter space. However, performing test simulations with 50000 particles shows that the lower resolution is sufficient for measuring the properties investigated here.

The simulation particles move initially on Keplerian orbits around the central star. The disc extends from an inner gap of $10 \mathrm{AU}$ to $100 \mathrm{AU}$. The inner cut-off avoids additional complex calculations of direct star-disc interactions and saves computation time. Any pseudo-particle that reaches a sphere of $1 \mathrm{AU}$ around the central star is removed from the simulation and stated in a commonly used simplified approach as having been accreted (Bate et al. 2002; Vorobyov \& Basu 2005; Pfalzner et al. 2008). The vertical density distribution in the disc follows

$\rho(r, z)=\rho_{0} \exp \left(-\frac{z^{2}}{2 H^{2}(r)}\right)$,

where $\rho_{0}$ is the unperturbed mid-plane particle density on the equatorial plane with $\rho_{0} \propto r^{-(b+1)}, H(r)$ is the vertical halfthickness of the disc (see Pringle 1981), which is represented by $H(r)=0.05 r$ according to a temperature profile of $T=T_{0} \cdot r^{-1}$ with $T=20 \mathrm{~K}$ at the inner edge of the disc.

The temporal development during the encounter is modelled using a fifth-order Runge-Kutta Cash-Karp integrator with an adaptive time-step size control for the numerical calculations. Long-range interactions of the gravitational forces between the disc particles and the perturbing star are calculated using a hierarchical tree method (Barnes \& Hut 1986).

The total simulation time of $t_{\text {end }} \approx 3000$ yrs for each encounter corresponds to three orbital periods of the outermost particles in the investigated standard disc with size $r_{\text {disc }}=100 \mathrm{AU}$ around a $1 M_{\odot}$ star. This time span was found to be adequate for the calculation of all relevant quantities.

The discs obtained at the end of the simulations continue to develop in the sense that taking into account viscous forces 
the eccentric orbits of perturbed disc pseudo-particles would recircularise on timescales probably in excess of $10^{5} \mathrm{yr}$. However, bound and unbound material can be clearly distinguished shortly after the encounter.

In this present study, we are interested in protoplanetary discs, which are usually of low mass compared to protostellar discs (Bate 2011). Here low-mass discs mean $m_{\text {disc }} \ll 0.1 M_{1}$, where $M_{1}$ is the mass of the disc surrounded star. The actual adopted disc-mass value is $10^{-4} M_{1}$. However, we tested another case of $m_{\text {disc }}=10^{-3} M_{1}$ and found no differences in the result when including self-gravity in the simulations. In addition, we performed test simulations including pressure and viscous forces using a SPH code (for a description of the code see Pfalzner $2003)$, but only found negligible differences $(<3 \%)$ in the results. Therefore, in most of our simulations self-gravitation and viscous forces were neglected in favour of higher computational performance (Pfalzner 2003, 2004).

The actual values of mass and angular momentum loss induced by the encounters were obtained by averaging over several simulations with different seeds for the initial particle distribution. The errors in the mass and angular momentum losses were determined as the maximum differences between the simulation results. They typically lie in the range of $2-3 \%$.

In addition to these obvious performance benefits, the possibility to neglect the self-gravitation, pressure, and viscous forces has allowed us to optimise the computation time by treating the disc particles as pseudo-particles without a fixed mass during the simulation. The standard method for generating the initial discmass distribution in the simulations is to assign each pseudoparticle the same mass (Boffin et al. 1998; Pfalzner 2004; Olczak et al. 2006; Pfalzner \& Olczak 2007). This approach involves the performance of a whole suite of simulations for each variation in the initial disc-mass distribution. In contrast, the different discmass distributions are realised by using a fixed pseudo-particle distribution and assigning their masses after the simulation process according to the desired density distribution in the disc. The implementation of this flexible numerical scheme allows us to use one suite of simulations for any initial disc-mass distribution. We note that the post-processing of the particle mass is only possible because of the restriction to low-mass discs, where selfgravitation and viscous forces can be neglected.

The particle masses are assigned to each pseudo-particle in the diagnostic step to calculate the encounter-induced losses and final mass distributions, which both depend on the initial distribution. For the example of a power-law disc-mass distribution with index $p$, the mass of a particle, $m_{\mathrm{i}}$, depends on its radial position in the disc $r_{i}$, the total disc mass $m_{\text {disc }}$, the number of pseudo-particles $n_{\text {part }}$, their radial positions $r_{j}$, and the underlying power-law particle distribution of index $b$. Since the number of pseudo-particles is limited, the discrete particle masses $m_{\mathrm{i}}$ are described by

$m_{\mathrm{i}}=\left(\sum_{j=1}^{n_{\text {part }}} r_{j}^{-p+b}\right)^{-1} \cdot m_{\mathrm{disc}} \cdot r_{\mathrm{i}}^{-p+b}$.

The resulting independence of the mass and the particle distributions allows us to improve the resolution of our simulations by placing most of the pseudo-particles initially where the interaction between the stars and disc is strongest.

In most of our simulations, we adopt a constant particle distribution $(b=0)$. This is done because the effects on mass and angular momentum are largest at the outskirts of the disc. Using a constant pseudo-particle density $(b=0)$ throughout the disc ensures a higher resolution in the outer parts, even for steep mass density profiles, than for the standard approach. In contrast, if one were interested in processes that mainly concern the inner parts of the disc, such as accretion, it would be more accurate to use not a constant particle distribution but one that guarantees a high resolution close to the star.

We tested the method of a posteriori mapping of the particle masses against representative test calculations for different initial pseudo-particle distributions and against the standard method. The differences in the results were negligible, thus justifying the generalisation of our results for a constant particle distribution $(b=0)$.

Apart from the density distribution, the outcome of an encounter depends on the relative periastron distance $r_{\text {peri }} / r_{\text {disc }}$ and the mass ratio of the involved stars $M_{2} / M_{1}$, where $M_{2}$ is the perturber mass. The parameter space that we investigated - for a summary see Table 1 - is chosen in such a way that it spans the entire range of encounters likely to occur in a typical young cluster, such as the Orion Nebula Cluster. Studies have shown that the ONC with its high central density might be typical of young clusters with a mass $>1000 M_{\odot}$ (Pfalzner 2009). The lower limit to the perturber mass ratio was chosen to be $M_{2} / M_{1}=0.1$ as for smaller mass ratios the influence of the perturber becomes insignificant. The upper limit, $M_{2} / M_{1}=500$, is determined by the maximum possible mass ratio in the ONC, which corresponds to the hydrogen burning limit, the lowest mass for which is $0.08 M_{\odot}$, and $40 M_{\odot}$, the approximate mass of the $\theta^{1} \mathrm{C}$ Ori system, which contains the most massive star in the ONC. The limit where perturbations at large distances become negligible depends on the perturber mass. The inner edge of the disc marks the lower value of $r_{\text {peri }} / r_{\text {disc }}=0.1$.

\section{Results}

Although in principle any mass distribution of circumstellar discs can be studied, four exemplary disc-mass distributions are investigated here in more detail. To cover the entire range of numerically and observationally determined disc-mass distributions, we consider a constant mass distribution ( $p=0)$, representing the lower boundary of expected distributions, and a $p=7 / 4$ distribution, providing an upper limit. Additionally, a $p=1$ distribution is investigated for comparison to previous star-disc encounter results and a $p=1 / 2$ distribution, which is in the range of analytical results for low-mass discs (Shakura \& Sunyaev 1973; Pringle 1981) and similar to results found in recent investigations of magnetised material (see Sect. 1).

The present investigation focuses on coplanar, prograde encounters on parabolic orbits $(e=1)$ (see example in Fig. 2a). Previous studies have shown that even for clusters as dense as the inner ONC region, most encounters in star clusters are expected to be close to parabolic (Larson 1990; Ostriker 1994; Olczak et al. 2010). These parabolic encounters provide the strongest impact of the perturber on the disc, since for higher eccentricities the perturber only interacts briefly with the star-disc system and is, therefore, unable to influence the disc significantly. The limitation to a certain orientation is more restricting, as inclined and retrograde encounters can lead to lower losses in mass (Heller 1993; Hall et al. 1996; Clarke \& Pringle 1993; Pfalzner et al. 2005b). However, Pfalzner et al. (2005b) showed that up to an inclination of $45^{\circ}$ the mass loss of the disc is only slightly smaller than for a coplanar orbit. This means that our results have to be regarded as an upper limit for encounters at a different inclination. 

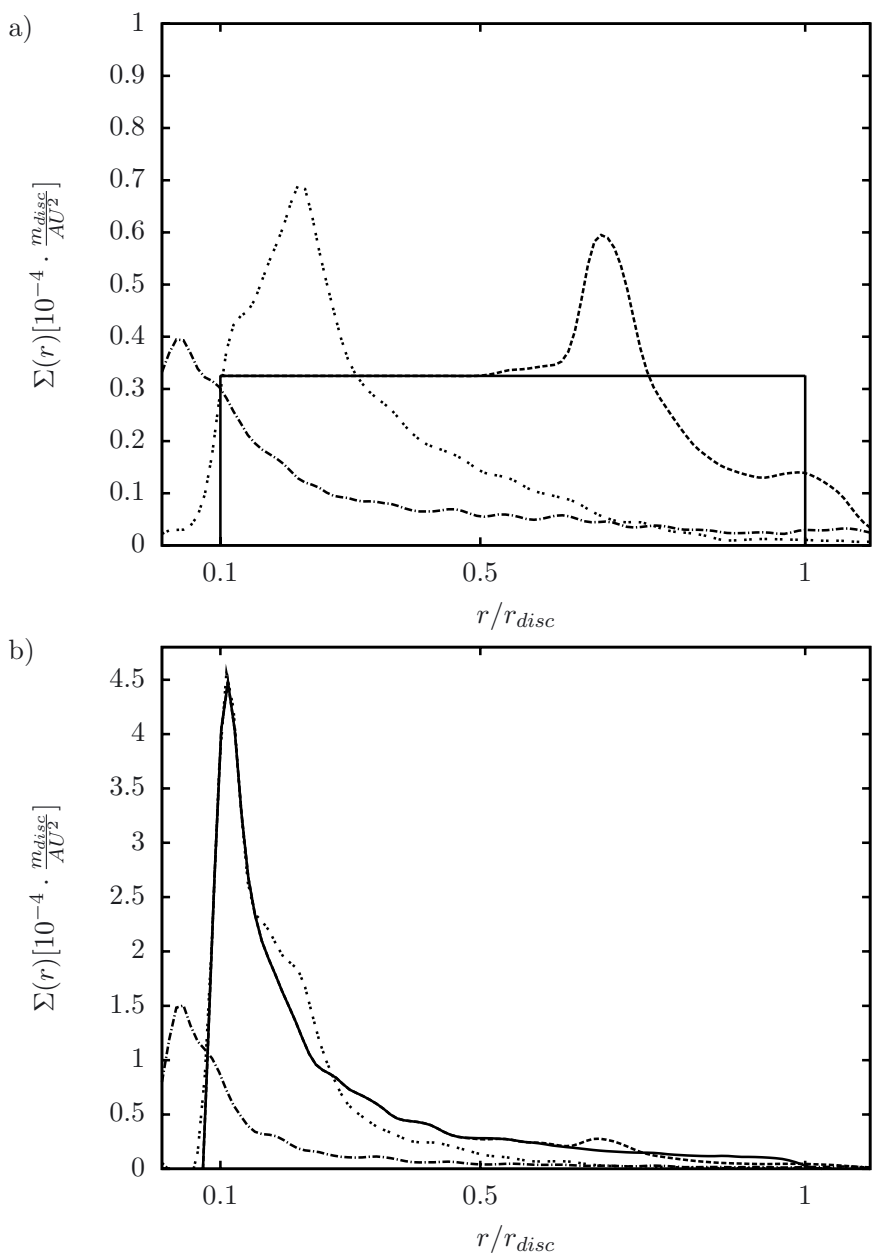

Fig. 1. Initial (solid line) and final surface densities in case of a) initially constant distributed disc material of $p=0$ and $\mathbf{b})$ a steep distribution of $p=7 / 4$. In both plots non-penetrating $\left(r_{\text {peri }} / r_{\text {disc }}=3\right.$, dashed line $)$, grazing $\left(r_{\text {peri }} / r_{\text {disc }}=1\right.$, double-dotted line $)$, and penetrating encounters $\left(r_{\text {peri }} / r_{\text {disc }}=0.1\right.$, dashed-dotted line $)$ are plotted for a perturbing star of equal mass $\left(M_{2} / M_{1}=1\right)$.

\subsection{Surface density distribution}

To achieve a clearer understanding of the re-distribution of the disc material during a star-disc encounter that depends on the initial disc-mass distribution, we first focus on the evolution of the surface density profiles. In Fig. 1, we present how the evolution of the surface density in star-disc encounters depends on the periastron distance for both a constant initial disc-mass distribution $(p=0)$ and one with a steep initial distribution ( $p=7 / 4)$. It shows the mass distributions before (solid line) and after a penetrating $\left(r_{\text {peri }} / r_{\text {disc }} \leq 1\right.$, dashed-dotted line $)$, grazing $\left(r_{\text {peri }} / r_{\text {disc }}=1\right.$, double-dotted line $)$, and distant encounter $\left(r_{\text {peri }} / r_{\text {disc }}=3\right.$, dashed line $)$. Here, the relative perturber mass was chosen to be $M_{2} / M_{1}=1$.

As known from previous investigations (Hall 1997; Larwood 1997), an encounter reduces the density in the outer parts of the disc by transporting some part of the outer disc material inwards and some part migrating outwards. The latter might become both captured by the perturber and unbound if the encounter is strong enough. These effects become more pronounced for small $r_{\text {peri }} / r_{\text {disc }}$ and/or large $M_{2} / M_{1}$. However, the actual amounts depend on the initial disc-mass distribution. In an equal-mass distant encounter $\left(r_{\text {peri }} / r_{\text {disc }}=3\right)$, most of the perturbed disc material is pushed inside the disc towards smaller disc radii. In this case, $12.5 \%$ of the total disc mass migrates inwards for an initial constant disc-mass distribution $(p=0)$, whereas it is only $6 \%$ for an initial $p=7 / 4$.

The effects of different initial disc-mass distributions becomes even more obvious for closer encounters such as grazing (double-dotted lines in Fig. 1) or penetrating fly-bys (dasheddotted lines in Fig. 1). Here, the migration process and also the differences between the initial distributions are dominated by material moving outwards. In the case of a grazing encounter and an initially constant disc-mass distribution, about $60 \%$ of the disc mass becomes unbound. However, in addition about $10 \%$ of the total disc mass can be found outside the initial disc radius of $100 \mathrm{AU}$ that is still bound to the central star. In contrast, for the steep $p=7 / 4$-mass distributions it only becomes $\sim 30 \%$ of the disc mass unbound but again $\sim 10 \%$ is bound but situated outside $100 \mathrm{AU}$. We conclude that the outer disc material is generally separated from the disc for grazing encounters, while material initially located inside the disc $\left(r / r_{\text {disc }} \leq 0.7\right)$ is re-distributed but remains bound to the central star. Hence, prominent differences in encounter-induced disc losses for the investigated disc-mass distributions are expected for strong perturbations of the outer disc parts. In penetrating encounters, part of the disc material is pushed further inside the disc resulting in a higher surface density in the inner disc regions. In extreme cases, the disc loss can increase to $>90 \%$ so that the final disc structure can no longer be regarded as a disc.

In nearly all cases, a steepening of the density profile is the general effect of an encounter. The degree of steepening depends on the mass ratio, periastron distance, and the initial disc-mass distribution. Figure 3 shows that even initially flat distributed disc-material $(p=0)$ can be redistributed into a surface density profile steeper than $p \approx 2$.

How is the vertical structure of the disc influenced by an encounter? As only coplanar encounters are investigated here, the answer is: surprisingly little. Figure $2 \mathrm{~b}$ shows the vertical particle profile of a strongly perturbed disc after a penetrating encounter of $M_{2} / M_{1}=1$. The main effect of the encounter is a decrease in the number of particles in the outer disc regions. In these parts of the disc, the resolution is relatively low, but owing to our constant particle distribution $(b=0)$ it is significantly higher than in previous investigations using $r^{-1}$ particle distributions in their simulations.

In a few special cases where material is pushed moderately inwards, even partially negative distribution indices as low as $p=-1$ can be the end product of an encounter (Fig. 4). The effect is most prominent in the inner part of perturbed discs with initially constant distributed disc material. Nonetheless, the outer parts of the disc always have a distribution with positive index, $p>0$. This could perhaps explain the negative indices observed for some discs. Observations remain limited to measuring only part of the radial extension of the entire disc. If this were to be the range where negative indices prevail, one would wrongly extrapolate negative indices for the whole disc, while the overall index of the disc would remain positive.

\subsection{Relative mass loss}

In addition to changing the shape of the disc-mass distributions, star-disc encounters can remove material from the disc. Figure 5 shows the relative disc-mass loss $\Delta m_{\text {rel }}=\left(m_{t=0}-m_{t_{\text {end }}}\right) / m_{t=0}$ for the four initial disc-mass distribution indices $p=0, p=$ $1 / 2, p=1$, and $p=7 / 4$. Two representative cases of orbital parameters are singled out: Fig. 5a shows the dependence on the periastron distance for an equal-mass encounter, whereas Fig. 5b 
a)

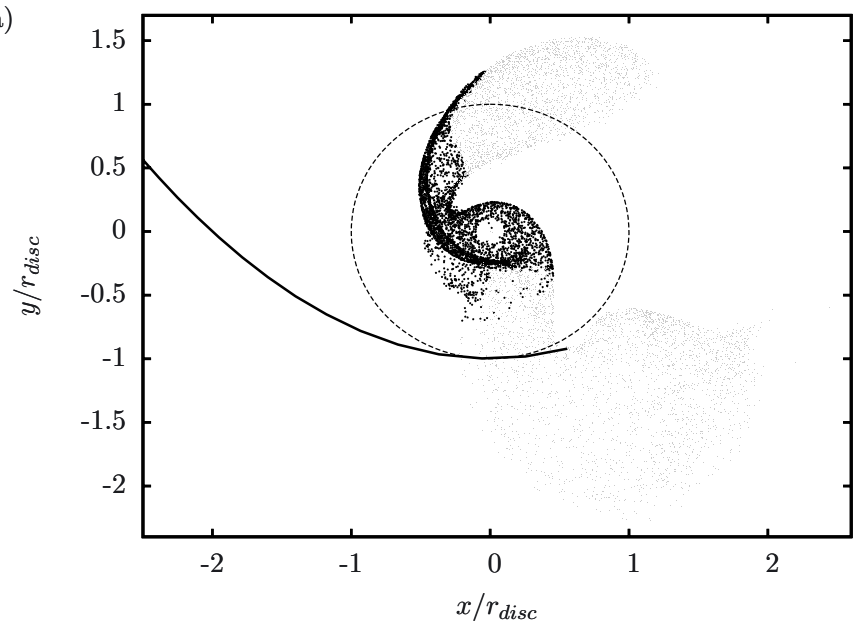

b)

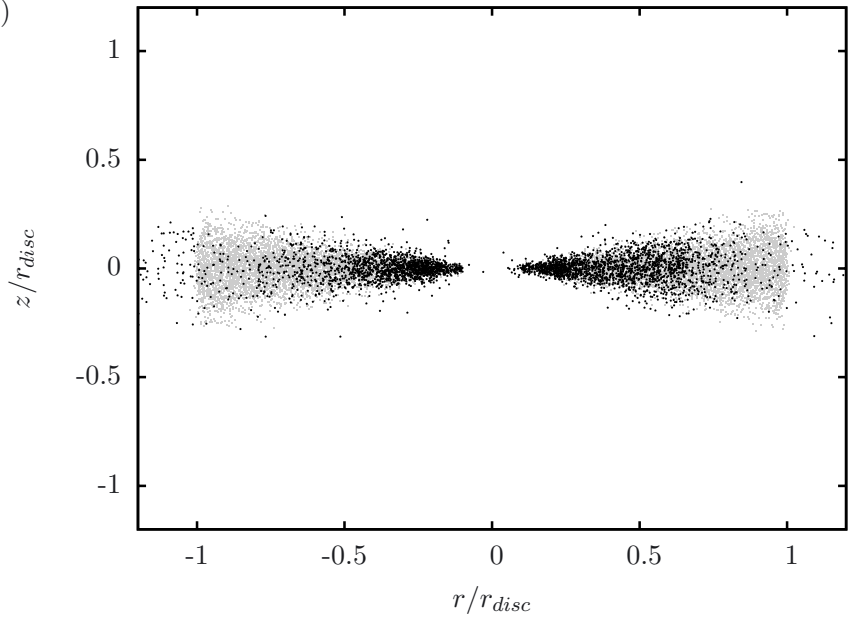

Fig. 2. The dashed line in a) shows the boundaries of the initial disc while the solid line indicates the trajectory of a grazing perturber $\left(r_{\text {peri }} / r_{\text {disc }}=1\right)$ of equal mass $\left(M_{2} / M_{1}=1\right)$. Material that resides within the disc after the perturbation is marked as black squares, while material that is in the end either bound to the perturbing star, unbound, or accreted is shown as grey dots. We note that the simulations were performed in three dimensions as can be seen in $\mathbf{b}$ ).

depicts the dependence on the mass ratio for grazing encounters $\left(r_{\text {peri }} / r_{\text {disc }}=1\right)$.

Qualitatively, the dependence of the relative disc-mass loss on the periastron distance agrees with previous results (Pfalzner et al. 2005b; Olczak et al. 2006). However, the absolute values change considerably for the different initial disc-mass distributions. The effect is largest for nearly grazing encounters where the outer disc regions are mainly affected. As expected from the respective fraction of material in the outer regions, maximal mass losses occur for initially constant disc-mass distributions, while minimal losses occur for the $r^{-7 / 4}$-distribution. The largest difference in mass loss between the investigated disc-mass distributions in Fig. 5a occurs for $r_{\text {peri }} / r_{\text {disc }}=0.9$, whereas the $r^{-7 / 4}$ distribution only has a mass loss of $33 \%$, and the constant mass distribution has a mass loss of $64 \%$.

The situation is somewhat different for very close penetrating encounters $\left(r_{\text {peri }} / r_{\text {disc }} \leq 0.3\right)$, where the discs are so strongly perturbed that the resulting structure can hardly be described as a disc. In this case the disc-mass loss seems relatively independent of the initial mass distribution (see Fig. 5a). At the other end of the parameter space - i.e. at large relative periastron distances

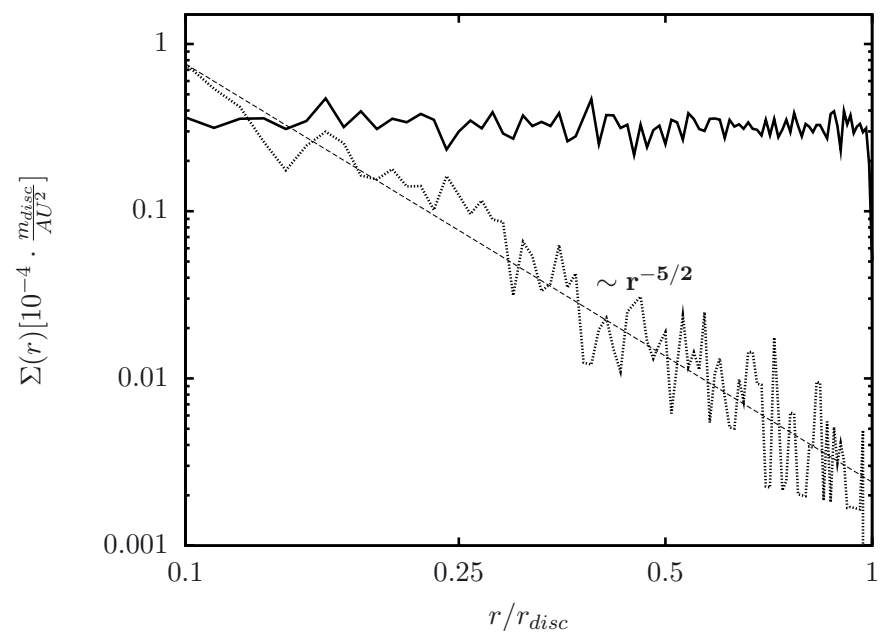

Fig. 3. Initial ( $p=0$, solid line) and final surface density after an encounter of $M_{2} / M_{1}=5.0$ and $r_{\text {peri }} / r_{\text {disc }}=0.7$ (dotted line). The dashed straight line represents a slope of $p=5 / 2$.

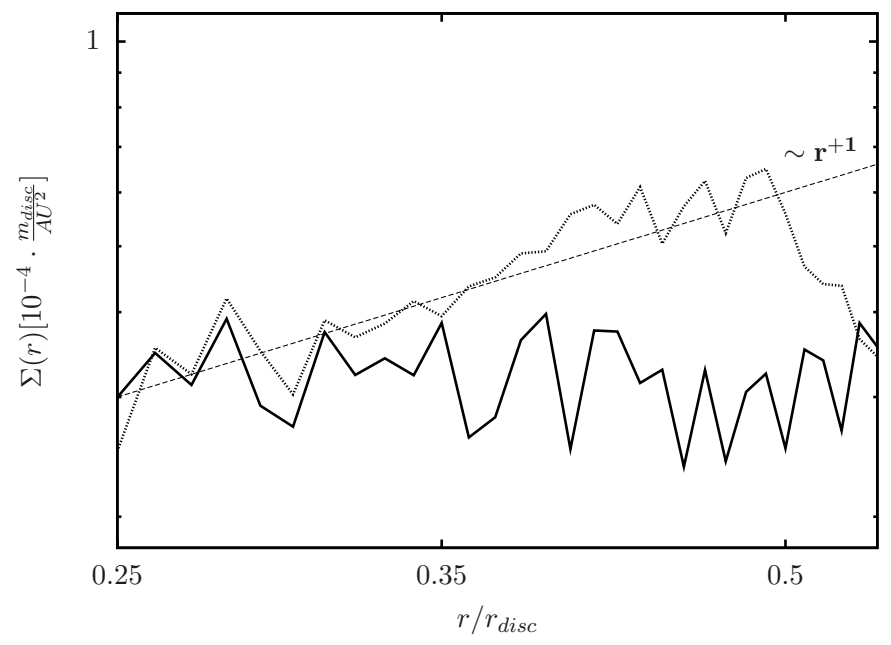

Fig. 4. Initial ( $p=0$, solid line) and final surface density after an encounter of $M_{2} / M_{1}=1.0$ and $r_{\text {peri }} / r_{\text {disc }}=2.0$ (dotted line). The dashed straight line represents a slope of $p=-1$.

- the mass loss becomes too small $\left(\Delta m_{\mathrm{rel}} \leq 10 \%\right)$ to infer any dependence on the initial distribution.

Similarly, the dependence on the initial mass distribution is less pronounced for $M_{2} / M_{1}<0.3$ and $M_{2} / M_{1}>90$ (see Fig. $5 b$ for a grazing encounter). Hence, generally weak perturbations whether distant or of low mass ratio $M_{2} / M_{1}$ - are incapable of significantly influencing the discs, while in the case of strong perturbations nearly the entire disc material is removed independently of the investigated disc-mass distributions. In both cases, the mass loss does not depend strongly on the disc-mass distribution. In contrast, encounters of intermediate strength are most sensitive to the disc-mass distribution. For the case shown in Fig. 5b, we find maximum differences of up to $35 \%$ for $M_{2} / M_{1}=3$.

For high-mass ratios $M_{2} / M_{1}>20$ and certain nonpenetrating periastron distances, which restrict the gravitational star-disc interactions to the outer disc parts, differences in mass loss as high as $40 \%$ can be inferred for the different initial discmass distributions (see Appendix A). 
a)

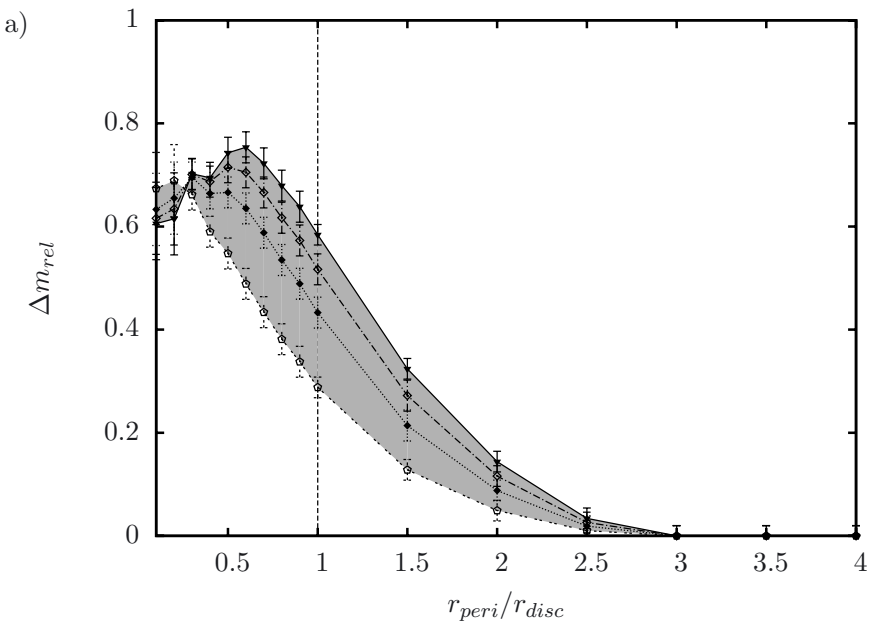

b)

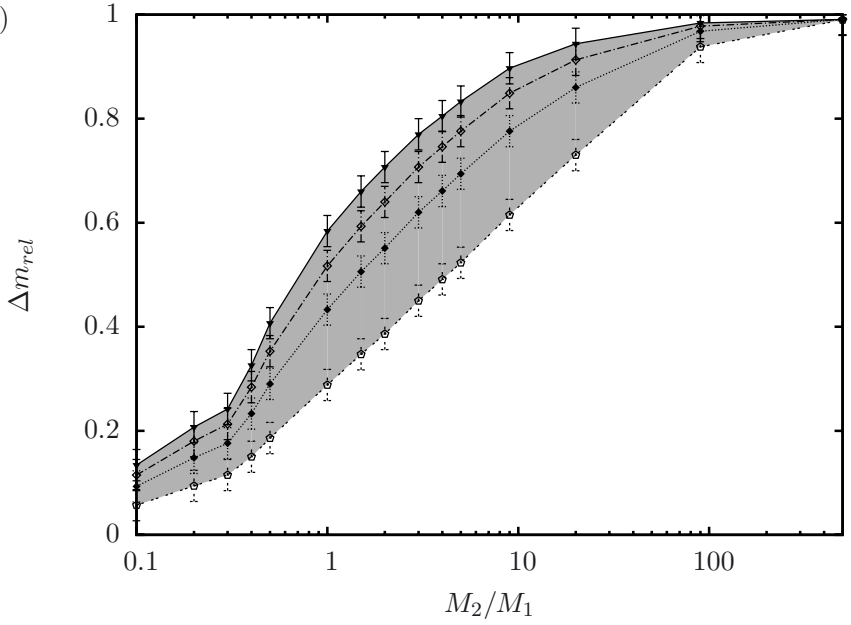

Fig. 5. The relative disc-mass loss of a $p=0$ (solid line), $p=1 / 2$ (dashed-dotted line), $p=1$ (dotted line), and $p=7 / 4$ (double-dotted line) disc-mass distribution including all particles bound more tightly to the central star than to the perturber and excluding unbound and accreted particles. The data is plotted for a) different periastron distances and an equal-mass perturber, and b) different perturber mass ratios and $r_{\text {peri }} / r_{\text {disc }}=1$. The vertical dashed line indicates the initial outer disc radius.

\subsection{Relative angular momentum loss}

The different initial disc-mass distributions do not only influence the disc-mass loss but also the angular momentum loss. Fig. 6a shows the relative angular momentum loss $\Delta J_{\text {rel }}=\left(J_{t=0}-\right.$ $\left.J_{t_{\text {end }}}\right) / J_{t=0}$ as a function of the encounter distance for equal-mass encounters and the four different disc-mass distributions used in this work. As expected from previous results (e.g. Pfalzner \& Olczak 2007), the general trends in relative angular momentum loss are quite similar to that of the mass loss (compare Figs. 5 and 6) with angular momentum losses being slightly higher than the disc-mass losses.

While the mass losses for encounters of intermediate strength with $r_{\text {peri }} / r_{\text {disc }}=0.9$ and $M_{2} / M_{1}=1$ are $64 \%$ for the constant mass distribution compared to $33 \%$ for the $7 / 4$-mass distribution as shown before, the corresponding angular momentum losses are $75 \%$ and $50 \%$, respectively. Material migrating inwards or becoming unbound owing to an encounter leads to an angular momentum loss, while the fraction of the disc mass that is pushed beyond the initial disc-radius but remains bound to the central star increases the total angular momentum of the disc. In total, the dependence on the initial disc-mass distributions is less
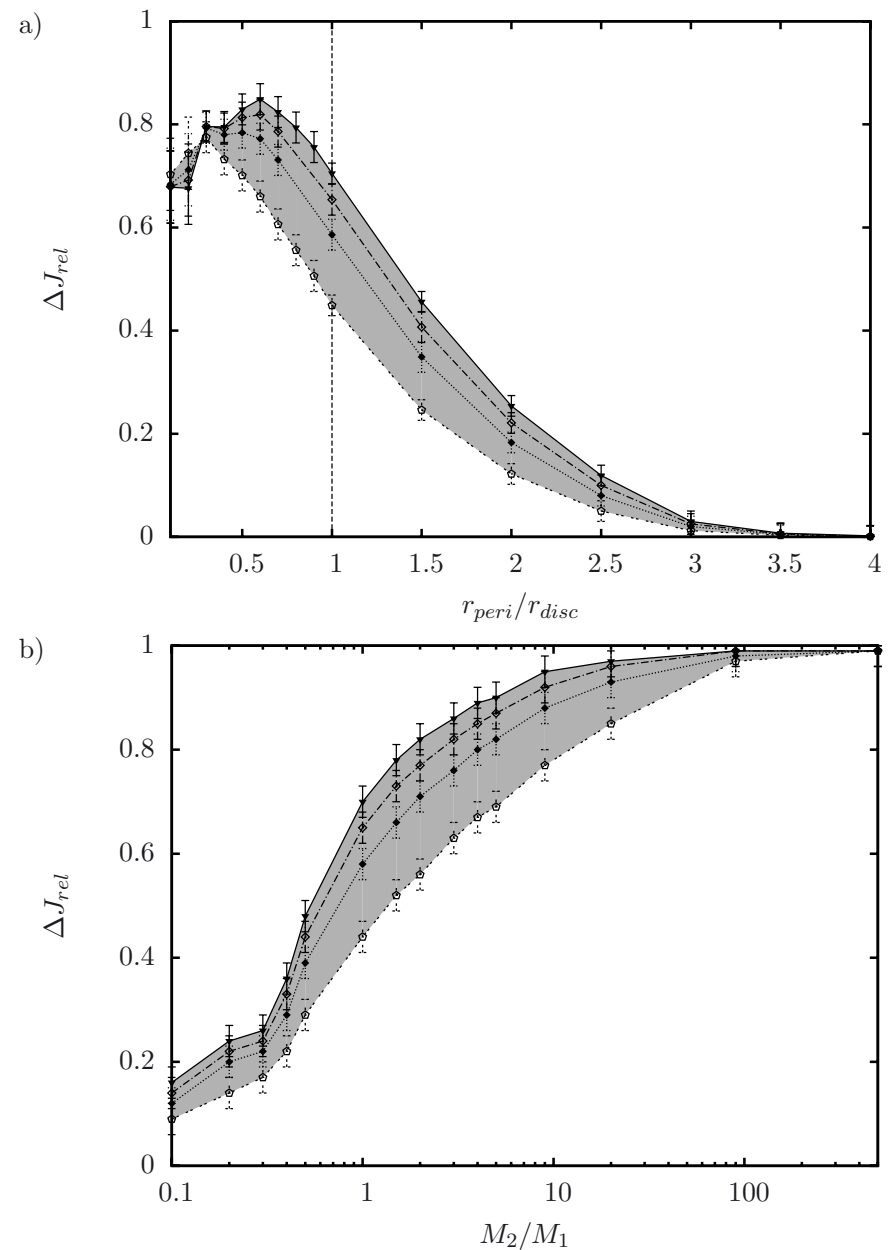

Fig. 6. The relative angular momentum loss of a $p=0$ (solid line), $p=1 / 2$ (dashed-dotted line), $p=1$ (dotted line), and $p=7 / 4$ (doubledotted line) disc-mass distribution including all particles bound more tightly to the central star than to the perturber and excluding unbound and accreted particles. The data is plotted for a) different periastron distances and an equal-mass perturber, and b) different perturber mass ratios and $r_{\text {peri }} / r_{\text {disc }}=1$. The vertical dashed line indicates the initial outer disc radius.

pronounced for the relative angular momentum loss than for the disc-mass loss.

However, although the differences are lower in maximum the influence of different initial disc-mass distributions on the angular momentum loss covers a significantly large parameter range (see also Appendix B).

Since mass and angular momentum losses are generally influenced by perturbations of the outer disc parts, an initially flat particle distribution that has a high resolution in the outer disc regions can help us to achieve a higher accuracy in determining disc losses. Nevertheless, disc losses obtained with initially steep particle distributions $(p=1)$ found in previous studies (Olczak et al. 2006; Pfalzner \& Olczak 2007) are accurately reproduced within the error range in the present study.

\subsection{Adapting a fit formula dependent on the initial disc-mass distribution}

The numerical results for the mass and angular momentum loss in this study cover a wide parameter range but, however, present only a discrete classification of the relative losses for the 
different initial disc-mass distributions. Analytical approaches are a possible option to avoid this disadvantage but are only valid for a very limited parameter space of distant encounters (Ostriker 1994; D’Onghia et al. 2010). To obtain a general estimate of the effect of arbitrary initial disc-mass distributions on the encounter-induced mass and angular momentum loss of protoplanetary discs, we present a fit formula that is valid for any initial disc-mass distribution, given that it can be expressed by a power law of the form $\Sigma(r) \propto r^{-p}$. For this purpose, we extended the fit function for the relative mass loss of Olczak et al. (2006, see Eq. (4) therein) and for the relative angular momentum loss of Pfalzner \& Olczak (2007) (see Eq. (1) therein), which are valid for a $r^{-1}$-distribution, towards arbitrary surface density distribution indices $0 \leq p \leq 7 / 4$

$$
\begin{aligned}
\Delta m_{\mathrm{rel}}= & \left(\frac{M_{2}}{M_{2}+0.5 M_{1}}\right)^{1.2} \ln \left[\left(3-\frac{p}{4}\right) \cdot\left(r_{p}\right)^{0.1}\right] \\
& \times \exp \left\{-\sqrt{\frac{M_{1}}{M_{2}+0.5 M_{1}}}\left[\left(r_{p}\right)^{2-p / 2}-0.5\right]\left(p+\left|\frac{1-p}{2}\right|\right)\right\} \\
\Delta J_{\mathrm{rel}}= & 1.02\left[\frac{M_{2}}{M_{\text {tot }}}+\left(\frac{1-p}{5}-\frac{\exp (p)}{100}\right)\right]^{0.5 \cdot r_{p}} \\
& \times \exp \left(-\sqrt{\frac{M_{1}\left(r_{p}-0.7 r_{p}^{0.5}\right)^{3}}{M_{2}}}\right)
\end{aligned}
$$

where $r_{p}=r_{\text {peri }} / r_{\text {disc }}$ is the relative periastron distance.

For most of the parameter space, the adopted functions fit the data well within the margin of error and extend Ostriker's analytical function for the angular momentum loss considerably. Larger deviations of the fit functions from the simulated losses occur only for high encounter mass ratios of $M_{2} / M_{1}>20$, which was also the case for the established fit functions of Pfalzner \& Olczak (2007) and Olczak et al. (2006). In the case of discpenetrating orbits and low-mass ratios, the disc losses can be affected by Lindblad and Corotation resonances that are located in the inner disc regions and cause moderate deviations of the fit function from the expected disc losses.

The extended fit functions provide a significant improvement to previous analytical and numerical results. They cover an extensive parameter space of the most reasonable initial disc-mass distributions and the most relevant orbital parameter ranges expected for interactions in star clusters of any age.

\section{Discussion and conclusion}

Most stars are believed to form in clusters (Lada \& Lada 2003) and probably undergo at least one encounter closer than $1000 \mathrm{AU}$ during the lifetime of their disc $\left(\sim 10^{6}\right.$ yrs $)$ (Scally \& Clarke 2001). Hence, encounters are likely to have a significant effect on the disc structure. The scope of this study has been to determine the role of the initial mass distribution in the disc in this context. A full parameter study of star-disc encounters, similar to those expected to occur in young dense star clusters such as the Orion Nebula Cluster, has been performed with different discmass distributions of the form $\Sigma(r) \propto r^{-p}, p \in[0,1 / 2,1,7 / 4]$, which cover the whole range of observed mass distributions in discs. The main results can be summarised as follows:

1. The relative disc-mass loss among the different initial density distributions differs by up to $40 \%$ for the same type of encounter. The largest differences are associated with strong perturbations of the outer disc edge, i.e. a grazing encounter in the case of equal stellar masses.
2. Although larger amounts of relative angular momentum than disc mass are lost due to a star-disc encounter, the dependence on the initial disc-mass distribution is less pronounced. Nevertheless, half of the parameter range for which the angular momentum loss occurs correspond to differences among the investigated distributions of more than $15 \%$.

3. The disc-mass and angular momentum losses caused by a parabolic encounter can be fitted by a function that depends on the perturber mass ratio, the relative periastron distance, and the index of the initial disc-mass distribution.

4. A steepening of the surface density slope is a general effect of an encounter for any initial disc-mass distribution and can result in distribution indices of $p>2$ even in the case of initially flat distributions.

In short, we have quantified the intuitive result that the flatter the mass distribution the stronger the change in the disc mass and angular momentum caused by encounters. This infers that the importance of encounters in young stellar clusters and their potential to trigger encounter-induced losses might be reconsidered depending on the dominant initial disc-mass distribution.

Another consequence of such an encounter is a change in the surface density of the disc on short timescales. In this context, the potentially most significant result of this study is that close encounters can provoke density profiles steeper than $\Sigma(r) \propto r^{-2}$ independently of the initial disc-mass distributions. Since these density profiles are claimed to be the prerequisite for forming a planetary system similar to the Solar System, encounters in the early history of the Solar System might have provided these conditions (Adams 2010). Desch (2007) analytically inferred an initial surface density profile of the solar nebula protoplanetary disc of roughly $\Sigma(r) \propto r^{-2.2}$ to form the present planets of the Solar System. As a possible way of forming this steep disc-mass density profile, he considered photoevaporation by an external massive star (see also Mitchell \& Stewart 2010). Kuchner (2004) found similar results for extrasolar planetary systems suggesting large disc-mass distribution indices of $p=2.0 \pm 0.5$.

Our results emphasise that these steep density profiles do not have to exist ab initio or be formed by photoevaporation processes but that even discs that initially have evenly distributed material can fulfil the requirements for the formation of a Solar System type planetary system in the inner disc regions after a close encounter. Additional evidence of such an encounter in the case of the early Solar System was given by distant Solar System objects on highly eccentric orbits, such as the transneptunian object Sedna, and also the sharp outer edge of the Solar System at $~ 50$ AU from the Sun (Ida et al. 1999; Allen et al. 2001; Morbidelli \& Levison 2004; Kenyon \& Bromley 2004).

On the other extreme, encounters cannot lead only to profile steepening but also profile flattening. Even the unexpected surface distribution profiles of $p<0$ observed by Isella et al. (2009) can be explained by the influence of a perturbing star. Non-penetrating encounters of a star-disc system with an initially flat distribution of disc material can easily lead to these density profiles of the inner disc regions. However, photoevaporation as another significant external impact on protoplanetary discs would have to be considered as an additional driver in these considerations.

Consequently, if all young stars were to start out with the same disc density structure, the influence of the cluster environment by means of encounters in the very early and dense phases of cluster evolution could account for the observed multitude of disc-mass density profiles. 


\section{References}

Adams, F. C. 2010, ARA\&A, 48, 47

Akeson, R. L., Ciardi, D. R., van Belle, G. T., \& Creech-Eakman, M. J. 2002, ApJ, 566, 1124

Alexander, R. D., Clarke, C. J., \& Pringle, J. E. 2005, MNRAS, 358, 283

Alexander, R. D., Clarke, C. J., \& Pringle, J. E. 2006, MNRAS, 369, 229

Allen, R. L., Bernstein, G. M., \& Malhotra, R. 2001, ApJ, 549, L241

Andrews, S. M., \& Williams, J. P. 2007a, ApJ, 671, 1800

Andrews, S. M., \& Williams, J. P. 2007b, ApJ, 659, 705

Balbus, S. A., \& Hawley, J. F. 2002, ApJ, 573, 749

Barnes, J., \& Hut, P. 1986, Nature, 324, 446

Bate, M. R. 2011, MNRAS, 1310

Bate, M. R., Bonnell, I. A., \& Bromm, V. 2002, MNRAS, 336, 705

Boffin, H. M. J., Watkins, S. J., Bhattal, A. S., Francis, N., \& Whitworth, A. P. 1998, MNRAS, 300, 1189

Clarke, C. J., \& Pringle, J. E. 1993, MNRAS, 261, 190

Clarke, C. J., Gendrin, A., \& Sotomayor, M. 2001, MNRAS, 328, 485

Currie, T., Kenyon, S. J., Balog, Z., et al. 2008, ApJ, 672, 558

Desch, S. J. 2007, ApJ, 671, 878

D’Onghia, E., Vogelsberger, M., Faucher-Giguere, C.-A., \& Hernquist, L. 2010, ApJ, 725, 353

Drake, J. J., Ercolano, B., Flaccomio, E., \& Micela, G. 2009, ApJ, 699, L35

Ercolano, B., Drake, J. J., Raymond, J. C., \& Clarke, C. C. 2008, ApJ, 688, 398

Gorti, U., \& Hollenbach, D. 2009, ApJ, 690, 1539

Haisch, Jr., K. E., Lada, E. A., \& Lada, C. J. 2001, ApJ, 553, L153

Hall, S. M. 1997, MNRAS, 287, 148

Hall, S. M., Clarke, C. J., \& Pringle, J. E. 1996, MNRAS, 278, 303

Hartmann, L., Calvet, N., Gullbring, E., \& D’Alessio, P. 1998, ApJ, 495, 385

Heller, C. H. 1993, ApJ, 408, 337

Heller, C. H. 1995, ApJ, 455, 252

Hernández, J., Calvet, N., Briceño, C., et al. 2007, ApJ, 671, 1784

Hernández, J., Hartmann, L., Calvet, N., et al. 2008, ApJ, 686, 1195

Hillenbrand, L. A. 2002 [arXiv: astro-ph/0210520]

Hueso, R., \& Guillot, T. 2005, A\&A, 442, 703

Ida, S., Larwood, J. D., \& Burkert, A. 1999, BAAS, 31, 1095

Isella, A., Carpenter, J. M., \& Sargent, A. I. 2009, ApJ, 701, 260

Johnstone, D., Matsuyama, I., McCarthy, I. G., \& Font, A. S. 2004, in Rev.

Mex. Astron. Astrofis. Conf. Ser. 22, ed. G. Garcia-Segura, G. Tenorio-Tagle,

J. Franco, \& H. W. Yorke, 38

Kenyon, S. J., \& Bromley, B. C. 2004, Nature, 432, 598

Kitamura, Y., Momose, M., Yokogawa, S., et al. 2002, ApJ, 581, 357
Klahr, H. H., \& Bodenheimer, P. 2003, ApJ, 582, 869

Kley, W., Papaloizou, J. C. B., \& Ogilvie, G. I. 2008, A\&A, 487, 671

Kuchner, M. J. 2004, ApJ, 612, 1147

Lada, C. J., \& Lada, E. A. 2003, ARA\&A, 41, 57

Lada, C. J., Muench, A. A., Haisch, Jr., K. E., et al. 2000, AJ, 120, 3162

Larson, R. B. 1990, in Physical Processes in Fragmentation and Star Formation, ed. R. Capuzzo-Dolcetta, C. Chiosi, \& A. di Fazio, Astrophys. Space Sci. Lib., 162, 461

Larwood, J. D. 1997, MNRAS, 290, 490

Lay, O. P., Carlstrom, J. E., \& Hills, R. E. 1997, ApJ, 489, 917

Lin, D. N. C., \& Pringle, J. E. 1990, ApJ, 358, 515

Mamajek, E. E. 2009, in AIP Conf. Ser. 1158, ed. T. Usuda, M. Tamura, \& M. Ishii, 3

Massi, F., di Carlo, E., Codella, C., et al. 2010, A\&A, 516, A52

Matsuyama, I., Johnstone, D., \& Hartmann, L. 2003, ApJ, 582, 893

Mitchell, T. R., \& Stewart, G. R. 2010, ApJ, 722, 1115

Moeckel, N., \& Bally, J. 2006, ApJ, 653, 437

Morbidelli, A., \& Levison, H. F. 2004, AJ, 128, 2564

Mundy, L. G., Looney, L. W., Erickson, W., et al. 1996, ApJ, 464, L169

Olczak, C., Pfalzner, S., \& Spurzem, R. 2006, ApJ, 642, 1140

Olczak, C., Pfalzner, S., \& Eckart, A. 2010, A\&A, 509, A63

Ostriker, E. C. 1994, ApJ, 424, 292

Pfalzner, S. 2003, ApJ, 592, 986

Pfalzner, S. 2004, ApJ, 602, 356

Pfalzner, S. 2009, A\&A, 498, L37

Pfalzner, S., \& Olczak, C. 2007, A\&A, 462, 193

Pfalzner, S., Umbreit, S., \& Henning, T. 2005a, ApJ, 629, 526

Pfalzner, S., Vogel, P., Scharwächter, J., \& Olczak, C. 2005b, A\&A, 437, 967

Pfalzner, S., Tackenberg, J., \& Steinhausen, M. 2008, A\&A, 487, L45

Pringle, J. E. 1981, ARA\&A, 19, 137

Scally, A., \& Clarke, C. 2001, MNRAS, 325, 449

Shakura, N. I., \& Sunyaev, R. A. 1973, A\&A, 24, 337

Shu, F. H., Adams, F. C., \& Lizano, S. 1987, ARA\&A, 25, 23

Shu, F. H., Galli, D., Lizano, S., Glassgold, A. E., \& Diamond, P. H. 2007, ApJ, 665,535

Sicilia-Aguilar, A., Hartmann, L., Calvet, N., et al. 2006, ApJ, 638, 897

Testi, L., Natta, A., Shepherd, D. S., \& Wilner, D. J. 2001, ApJ, 554, 1087

Vorobyov, E. I., \& Basu, S. 2005, ApJ, 633, L137

Vorobyov, E. I., \& Basu, S. 2007, MNRAS, 381, 1009

Wilner, D. J., Ho, P. T. P., Kastner, J. H., \& Rodríguez, L. F. 2000, ApJ, 534, L101

Pages 9 to 16 are available in the electronic edition of the journal at http://www . aanda. org 
M. Steinhausen et al.: Disc-mass distribution in star-disc encounters

Appendix A: Relative mass loss

Table A.1. Relative disc-mass loss assuming an initial disc-mass distribution of $p=0$.

\begin{tabular}{|c|c|c|c|c|c|c|c|c|c|c|c|c|c|c|}
\hline & $0.1 M_{1}$ & $0.3 M_{1}$ & $0.5 M_{1}$ & $1 M_{1}$ & $1.5 M_{1}$ & $2 M_{1}$ & $3 M_{1}$ & $4 M_{1}$ & $5 M_{1}$ & $9 M_{1}$ & $20 M_{1}$ & $50 M_{1}$ & $90 M_{1}$ & $500 M_{1}$ \\
\hline 00.1 & 0.16 & 0.42 & 0.59 & 0.61 & 0.61 & 0.61 & 0.64 & 0.67 & 0.74 & 0.84 & 0.88 & 0.85 & 0.87 & 0.91 \\
\hline 00.2 & 0.16 & 0.41 & 0.56 & 0.62 & 0.75 & 0.80 & 0.82 & 0.83 & 0.86 & 0.90 & 0.91 & 0.90 & 0.92 & 0.93 \\
\hline 00.3 & 0.15 & 0.39 & 0.55 & 0.70 & 0.79 & 0.83 & 0.86 & 0.90 & 0.92 & 0.94 & 0.95 & 0.98 & 0.98 & 0.98 \\
\hline 00.4 & 0.15 & 0.32 & 0.53 & 0.69 & 0.81 & 0.86 & 0.92 & 0.95 & 0.95 & 0.97 & 0.98 & 0.98 & 0.99 & 0.99 \\
\hline 00.5 & 0.15 & 0.28 & 0.53 & 0.73 & 0.83 & 0.87 & 0.91 & 0.93 & 0.95 & 0.97 & 0.98 & 0.99 & 0.99 & 0.99 \\
\hline 00.6 & 0.16 & 0.27 & 0.55 & 0.75 & 0.81 & 0.84 & 0.89 & 0.91 & 0.93 & 0.97 & 0.98 & 0.99 & 0.99 & 0.99 \\
\hline 00.7 & 0.15 & 0.26 & 0.55 & 0.72 & 0.78 & 0.81 & 0.86 & 0.89 & 0.91 & 0.94 & 0.97 & 0.99 & 0.99 & 0.99 \\
\hline 00.8 & 0.15 & 0.26 & 0.51 & 0.68 & 0.75 & 0.78 & 0.83 & 0.86 & 0.89 & 0.94 & 0.97 & 0.98 & 0.99 & 0.99 \\
\hline 00.9 & 0.14 & 0.26 & 0.46 & 0.64 & 0.70 & 0.75 & 0.80 & 0.84 & 0.86 & 0.91 & 0.96 & 0.98 & 0.99 & 0.99 \\
\hline 01.0 & 0.13 & 0.24 & 0.41 & 0.58 & 0.66 & 0.71 & 0.77 & 0.81 & 0.83 & 0.90 & 0.94 & 0.97 & 0.98 & 0.99 \\
\hline 01.5 & 0.04 & 0.14 & 0.19 & 0.32 & 0.44 & 0.49 & 0.59 & 0.65 & 0.69 & 0.78 & 0.88 & 0.94 & 0.96 & 0.99 \\
\hline 02.0 & 0.00 & 0.03 & 0.07 & 0.14 & 0.20 & 0.29 & 0.39 & 0.46 & 0.52 & 0.64 & 0.79 & 0.89 & 0.93 & 0.98 \\
\hline 02.5 & 0.00 & 0.00 & 0.00 & 0.03 & 0.09 & 0.14 & 0.23 & 0.30 & 0.35 & 0.50 & 0.68 & 0.82 & 0.89 & 0.96 \\
\hline 03.0 & 0.00 & 0.00 & 0.00 & 0.00 & 0.02 & 0.04 & 0.09 & 0.14 & 0.19 & 0.36 & 0.56 & 0.76 & 0.84 & 0.95 \\
\hline 03.5 & 0.00 & 0.00 & 0.00 & 0.00 & 0.00 & 0.00 & 0.03 & 0.06 & 0.10 & 0.23 & 0.44 & 0.68 & 0.78 & 0.93 \\
\hline 04.0 & 0.00 & 0.00 & 0.00 & 0.00 & 0.00 & 0.00 & 0.00 & 0.01 & 0.02 & 0.14 & 0.34 & 0.58 & 0.72 & 0.90 \\
\hline 04.5 & 0.00 & 0.00 & 0.00 & 0.00 & 0.00 & 0.00 & 0.00 & 0.00 & 0.00 & 0.05 & 0.23 & 0.49 & 0.64 & 0.89 \\
\hline 05.0 & 0.0 & 0.00 & 0.0 & 0.00 & 0.00 & 0.00 & 0.00 & 0.00 & 0.00 & 0.02 & 0.17 & 0.41 & 0.58 & 0.86 \\
\hline 05.5 & 0.0 & 0.0 & 0.0 & 0.0 & 0.00 & 0.00 & 0.00 & 0.00 & 0.00 & 0.00 & 0.08 & 0.32 & 0.48 & 0.83 \\
\hline 06.0 & 0.0 & 0.0 & 0.0 & 0.0 & 0.00 & 0.00 & 0.00 & 0.00 & 0.00 & 0.00 & 0.05 & 0.26 & 0.41 & 0.80 \\
\hline 06.5 & 0.0 & 0.0 & 0.0 & 0.0 & 0.0 & 0.00 & 0.00 & 0.00 & 0.00 & 0.00 & 0.02 & 0.18 & 0.35 & 0.76 \\
\hline 07.0 & 0.0 & 0.0 & 0.0 & 0.0 & 0.0 & 0.00 & 0.00 & 0.00 & 0.00 & 0.00 & 0.00 & 0.15 & 0.27 & 0.72 \\
\hline 07.5 & 0.0 & 0.0 & 0.0 & 0.0 & 0.0 & 0.0 & 0.0 & 0.0 & 0.00 & 0.00 & 0.00 & 0.08 & 0.23 & 0.69 \\
\hline 08.0 & 0.0 & 0.0 & 0.0 & 0.0 & 0.0 & 0.0 & 0.0 & 0.0 & 0.00 & 0.00 & 0.00 & 0.06 & 0.20 & 0.65 \\
\hline 08.5 & 0.0 & 0.0 & 0.0 & 0.0 & 0.0 & 0.0 & 0.0 & 0.0 & 0.0 & 0.00 & 0.00 & 0.01 & 0.14 & 0.61 \\
\hline 09.0 & 0.0 & 0.0 & 0.0 & 0.0 & 0.0 & 0.0 & 0.0 & 0.0 & 0.0 & 0.00 & 0.00 & 0.01 & 0.12 & 0.56 \\
\hline 09.5 & 0.0 & 0.0 & 0.0 & 0.0 & 0.0 & 0.0 & 0.0 & 0.0 & 0.0 & 0.0 & 0.00 & 0.00 & 0.06 & 0.52 \\
\hline 10.0 & 0.0 & 0.0 & 0.0 & 0.0 & 0.0 & 0.0 & 0.0 & 0.0 & 0.0 & 0.0 & 0.00 & 0.00 & 0.05 & 0.46 \\
\hline 10.5 & 0.0 & 0.0 & 0.0 & 0.0 & 0.0 & 0.0 & 0.0 & 0.0 & 0.0 & 0.0 & 0.0 & 0.00 & 0.01 & 0.43 \\
\hline 11.0 & 0.0 & 0.0 & 0.0 & 0.0 & 0.0 & 0.0 & 0.0 & 0.0 & 0.0 & 0.0 & 0.0 & 0.00 & 0.01 & 0.38 \\
\hline 11.5 & 0.0 & 0.0 & 0.0 & 0.0 & 0.0 & 0.0 & 0.0 & 0.0 & 0.0 & 0.0 & 0.0 & 0.00 & 0.00 & 0.35 \\
\hline 12.0 & 0.0 & 0.0 & 0.0 & 0.0 & 0.0 & 0.0 & 0.0 & 0.0 & 0.0 & 0.0 & 0.0 & 0.00 & 0.00 & 0.30 \\
\hline 12.5 & 0.0 & 0.0 & 0.0 & 0.0 & 0.0 & 0.0 & 0.0 & 0.0 & 0.0 & 0.0 & 0.0 & 0.00 & 0.00 & 0.28 \\
\hline 13.0 & 0.0 & 0.0 & 0.0 & 0.0 & 0.0 & 0.0 & 0.0 & 0.0 & 0.0 & 0.0 & 0.0 & 0.00 & 0.00 & 0.23 \\
\hline 13.5 & 0.0 & 0.0 & 0.0 & 0.0 & 0.0 & 0.0 & 0.0 & 0.0 & 0.0 & 0.0 & 0.0 & 0.00 & 0.00 & 0.22 \\
\hline 14.0 & 0.0 & 0.0 & 0.0 & 0.0 & 0.0 & 0.0 & 0.0 & 0.0 & 0.0 & 0.0 & 0.0 & 0.00 & 0.00 & 0.17 \\
\hline 14.5 & 0.0 & 0.0 & 0.0 & 0.0 & 0.0 & 0.0 & 0.0 & 0.0 & 0.0 & 0.0 & 0.0 & 0.00 & 0.00 & 0.17 \\
\hline 15.0 & 0.0 & 0.0 & 0.0 & 0.0 & 0.0 & 0.0 & 0.0 & 0.0 & 0.0 & 0.0 & 0.0 & 0.00 & 0.00 & 0.12 \\
\hline 15.5 & 0.0 & 0.0 & 0.0 & 0.0 & 0.0 & 0.0 & 0.0 & 0.0 & 0.0 & 0.0 & 0.0 & 0.0 & 0.00 & 0.12 \\
\hline 16.0 & 0.0 & 0.0 & 0.0 & 0.0 & 0.0 & 0.0 & 0.0 & 0.0 & 0.0 & 0.0 & 0.0 & 0.0 & 0.00 & 0.12 \\
\hline 16.5 & 0.0 & 0.0 & 0.0 & 0.0 & 0.0 & 0.0 & 0.0 & 0.0 & 0.0 & 0.0 & 0.0 & 0.0 & 0.00 & 0.08 \\
\hline 17.0 & 0.0 & 0.0 & 0.0 & 0.0 & 0.0 & 0.0 & 0.0 & 0.0 & 0.0 & 0.0 & 0.0 & 0.0 & 0.00 & 0.08 \\
\hline 17.5 & 0.0 & 0.0 & 0.0 & 0.0 & 0.0 & 0.0 & 0.0 & 0.0 & 0.0 & 0.0 & 0.0 & 0.0 & 0.0 & 0.04 \\
\hline 18.0 & 0.0 & 0.0 & 0.0 & 0.0 & 0.0 & 0.0 & 0.0 & 0.0 & 0.0 & 0.0 & 0.0 & 0.0 & 0.0 & 0.04 \\
\hline 18.5 & 0.0 & 0.0 & 0.0 & 0.0 & 0.0 & 0.0 & 0.0 & 0.0 & 0.0 & 0.0 & 0.0 & 0.0 & 0.0 & 0.01 \\
\hline 19.0 & 0.0 & 0.0 & 0.0 & 0.0 & 0.0 & 0.0 & 0.0 & 0.0 & 0.0 & 0.0 & 0.0 & 0.0 & 0.0 & 0.01 \\
\hline 19.5 & 0.0 & 0.0 & 0.0 & 0.0 & 0.0 & 0.0 & 0.0 & 0.0 & 0.0 & 0.0 & 0.0 & 0.0 & 0.0 & 0.00 \\
\hline 20.0 & 0.0 & 0.0 & 0.0 & 0.0 & 0.0 & 0.0 & 0.0 & 0.0 & 0.0 & 0.0 & 0.0 & 0.0 & 0.0 & 0.00 \\
\hline
\end{tabular}

Notes. The relative disc-mass loss considering particles bound tighter to the central star than to the encounter for all simulated configurations of prograde parabolic $(e=1)$ star-disc encounters. The first row contains the perturber masses $M_{2}$ in units of $M_{1}$, the first column contains the relative periastra $r_{\text {peri }} / r_{\text {disc }}$. 
Table A.2. Relative disc-mass loss assuming an initial disc-mass distribution of $p=1 / 2$.

\begin{tabular}{|c|c|c|c|c|c|c|c|c|c|c|c|c|c|c|}
\hline & $0.1 M_{1}$ & $0.3 M_{1}$ & $0.5 M_{1}$ & $1 M_{1}$ & $1.5 M_{1}$ & $2 M_{1}$ & $3 M_{1}$ & $4 M_{1}$ & $5 M_{1}$ & $9 M_{1}$ & $20 M_{1}$ & $50 M_{1}$ & $90 M_{1}$ & $500 M_{1}$ \\
\hline 00.1 & 0.16 & 0.42 & 0.59 & 0.61 & 0.64 & 0.64 & 0.67 & 0.70 & 0.76 & 0.85 & 0.89 & 0.86 & 0.88 & 0.91 \\
\hline 00.2 & 0.16 & 0.40 & 0.56 & 0.63 & 0.76 & 0.81 & 0.84 & 0.85 & 0.87 & 0.91 & 0.92 & 0.91 & 0.93 & 0.94 \\
\hline 00.3 & 0.15 & 0.37 & 0.54 & 0.70 & 0.79 & 0.83 & 0.87 & 0.90 & 0.93 & 0.95 & 0.96 & 0.98 & 0.99 & 0.98 \\
\hline 00.4 & 0.15 & 0.31 & 0.51 & 0.68 & 0.80 & 0.85 & 0.91 & 0.94 & 0.94 & 0.96 & 0.98 & 0.99 & 0.99 & 0.99 \\
\hline 00.5 & 0.15 & 0.27 & 0.51 & 0.70 & 0.79 & 0.84 & 0.89 & 0.91 & 0.93 & 0.96 & 0.98 & 0.99 & 0.99 & 0.99 \\
\hline 00.6 & 0.14 & 0.26 & 0.51 & 0.70 & 0.76 & 0.80 & 0.86 & 0.88 & 0.90 & 0.95 & 0.98 & 0.99 & 0.99 & 0.99 \\
\hline 00.7 & 0.14 & 0.24 & 0.50 & 0.66 & 0.73 & 0.76 & 0.81 & 0.85 & 0.87 & 0.92 & 0.96 & 0.99 & 0.99 & 0.99 \\
\hline 00.8 & 0.13 & 0.24 & 0.45 & 0.62 & 0.69 & 0.72 & 0.78 & 0.81 & 0.84 & 0.90 & 0.95 & 0.98 & 0.99 & 0.99 \\
\hline 00.9 & 0.12 & 0.23 & 0.40 & 0.57 & 0.63 & 0.69 & 0.74 & 0.78 & 0.81 & 0.87 & 0.93 & 0.97 & 0.98 & 0.99 \\
\hline 01.0 & 0.12 & 0.21 & 0.35 & 0.52 & 0.59 & 0.64 & 0.71 & 0.75 & 0.78 & 0.85 & 0.91 & 0.96 & 0.98 & 0.99 \\
\hline 01.5 & 0.03 & 0.11 & 0.15 & 0.27 & 0.37 & 0.43 & 0.51 & 0.57 & 0.61 & 0.72 & 0.83 & 0.90 & 0.93 & 0.99 \\
\hline 02.0 & 0.00 & 0.02 & 0.05 & 0.12 & 0.17 & 0.24 & 0.32 & 0.39 & 0.44 & 0.56 & 0.72 & 0.83 & 0.89 & 0.97 \\
\hline 02.5 & 0.00 & 0.00 & 0.00 & 0.03 & 0.07 & 0.11 & 0.19 & 0.24 & 0.29 & 0.43 & 0.60 & 0.75 & 0.83 & 0.94 \\
\hline 03.0 & 0.00 & 0.00 & 0.00 & 0.00 & 0.02 & 0.03 & 0.07 & 0.11 & 0.15 & 0.30 & 0.48 & 0.68 & 0.77 & 0.92 \\
\hline 03.5 & 0.00 & 0.00 & 0.00 & 0.00 & 0.00 & 0.00 & 0.02 & 0.05 & 0.08 & 0.18 & 0.37 & 0.60 & 0.70 & 0.89 \\
\hline 04.0 & 0.00 & 0.00 & 0.00 & 0.00 & 0.00 & 0.00 & 0.00 & 0.00 & 0.02 & 0.11 & 0.28 & 0.50 & 0.63 & 0.85 \\
\hline 04.5 & 0.00 & 0.00 & 0.00 & 0.00 & 0.00 & 0.00 & 0.00 & 0.00 & 0.00 & 0.04 & 0.18 & 0.41 & 0.56 & 0.83 \\
\hline 05.0 & 0.0 & 0.00 & 0.0 & 0.00 & 0.00 & 0.00 & 0.00 & 0.00 & 0.00 & 0.01 & 0.13 & 0.34 & 0.49 & 0.79 \\
\hline 05.5 & 0.0 & 0.0 & 0.0 & 0.0 & 0.00 & 0.00 & 0.00 & 0.00 & 0.00 & 0.00 & 0.06 & 0.26 & 0.40 & 0.76 \\
\hline 06.0 & 0.0 & 0.0 & 0.0 & 0.0 & 0.00 & 0.00 & 0.00 & 0.00 & 0.00 & 0.00 & 0.04 & 0.21 & 0.34 & 0.72 \\
\hline 06.5 & 0.0 & 0.0 & 0.0 & 0.0 & 0.0 & 0.00 & 0.00 & 0.00 & 0.00 & 0.00 & 0.02 & 0.14 & 0.29 & 0.68 \\
\hline 07.0 & 0.0 & 0.0 & 0.0 & 0.0 & 0.0 & 0.00 & 0.00 & 0.00 & 0.00 & 0.00 & 0.00 & 0.12 & 0.21 & 0.64 \\
\hline 07.5 & 0.0 & 0.0 & 0.0 & 0.0 & 0.0 & 0.0 & 0.0 & 0.0 & 0.00 & 0.00 & 0.00 & 0.06 & 0.18 & 0.61 \\
\hline 08.0 & 0.0 & 0.0 & 0.0 & 0.0 & 0.0 & 0.0 & 0.0 & 0.0 & 0.00 & 0.00 & 0.00 & 0.05 & 0.16 & 0.56 \\
\hline 08.5 & 0.0 & 0.0 & 0.0 & 0.0 & 0.0 & 0.0 & 0.0 & 0.0 & 0.0 & 0.00 & 0.00 & 0.01 & 0.11 & 0.53 \\
\hline 09.0 & 0.0 & 0.0 & 0.0 & 0.0 & 0.0 & 0.0 & 0.0 & 0.0 & 0.0 & 0.00 & 0.00 & 0.00 & 0.09 & 0.47 \\
\hline 09.5 & 0.0 & 0.0 & 0.0 & 0.0 & 0.0 & 0.0 & 0.0 & 0.0 & 0.0 & 0.0 & 0.00 & 0.00 & 0.05 & 0.44 \\
\hline 10.0 & 0.0 & 0.0 & 0.0 & 0.0 & 0.0 & 0.0 & 0.0 & 0.0 & 0.0 & 0.0 & 0.00 & 0.00 & 0.04 & 0.38 \\
\hline 10.5 & 0.0 & 0.0 & 0.0 & 0.0 & 0.0 & 0.0 & 0.0 & 0.0 & 0.0 & 0.0 & 0.0 & 0.00 & 0.01 & 0.35 \\
\hline 11.0 & 0.0 & 0.0 & 0.0 & 0.0 & 0.0 & 0.0 & 0.0 & 0.0 & 0.0 & 0.0 & 0.0 & 0.00 & 0.00 & 0.31 \\
\hline 11.5 & 0.0 & 0.0 & 0.0 & 0.0 & 0.0 & 0.0 & 0.0 & 0.0 & 0.0 & 0.0 & 0.0 & 0.00 & 0.00 & 0.29 \\
\hline 12.0 & 0.0 & 0.0 & 0.0 & 0.0 & 0.0 & 0.0 & 0.0 & 0.0 & 0.0 & 0.0 & 0.0 & 0.00 & 0.00 & 0.25 \\
\hline 12.5 & 0.0 & 0.0 & 0.0 & 0.0 & 0.0 & 0.0 & 0.0 & 0.0 & 0.0 & 0.0 & 0.0 & 0.00 & 0.00 & 0.23 \\
\hline 13.0 & 0.0 & 0.0 & 0.0 & 0.0 & 0.0 & 0.0 & 0.0 & 0.0 & 0.0 & 0.0 & 0.0 & 0.00 & 0.00 & 0.19 \\
\hline 13.5 & 0.0 & 0.0 & 0.0 & 0.0 & 0.0 & 0.0 & 0.0 & 0.0 & 0.0 & 0.0 & 0.0 & 0.00 & 0.00 & 0.18 \\
\hline 14.0 & 0.0 & 0.0 & 0.0 & 0.0 & 0.0 & 0.0 & 0.0 & 0.0 & 0.0 & 0.0 & 0.0 & 0.00 & 0.00 & 0.14 \\
\hline 14.5 & 0.0 & 0.0 & 0.0 & 0.0 & 0.0 & 0.0 & 0.0 & 0.0 & 0.0 & 0.0 & 0.0 & 0.00 & 0.00 & 0.14 \\
\hline 15.0 & 0.0 & 0.0 & 0.0 & 0.0 & 0.0 & 0.0 & 0.0 & 0.0 & 0.0 & 0.0 & 0.0 & 0.00 & 0.00 & 0.10 \\
\hline 15.5 & 0.0 & 0.0 & 0.0 & 0.0 & 0.0 & 0.0 & 0.0 & 0.0 & 0.0 & 0.0 & 0.0 & 0.0 & 0.00 & 0.10 \\
\hline 16.0 & 0.0 & 0.0 & 0.0 & 0.0 & 0.0 & 0.0 & 0.0 & 0.0 & 0.0 & 0.0 & 0.0 & 0.0 & 0.00 & 0.09 \\
\hline 16.5 & 0.0 & 0.0 & 0.0 & 0.0 & 0.0 & 0.0 & 0.0 & 0.0 & 0.0 & 0.0 & 0.0 & 0.0 & 0.00 & 0.06 \\
\hline 17.0 & 0.0 & 0.0 & 0.0 & 0.0 & 0.0 & 0.0 & 0.0 & 0.0 & 0.0 & 0.0 & 0.0 & 0.0 & 0.00 & 0.06 \\
\hline 17.5 & 0.0 & 0.0 & 0.0 & 0.0 & 0.0 & 0.0 & 0.0 & 0.0 & 0.0 & 0.0 & 0.0 & 0.0 & 0.0 & 0.03 \\
\hline 18.0 & 0.0 & 0.0 & 0.0 & 0.0 & 0.0 & 0.0 & 0.0 & 0.0 & 0.0 & 0.0 & 0.0 & 0.0 & 0.0 & 0.03 \\
\hline 18.5 & 0.0 & 0.0 & 0.0 & 0.0 & 0.0 & 0.0 & 0.0 & 0.0 & 0.0 & 0.0 & 0.0 & 0.0 & 0.0 & 0.01 \\
\hline 19.0 & 0.0 & 0.0 & 0.0 & 0.0 & 0.0 & 0.0 & 0.0 & 0.0 & 0.0 & 0.0 & 0.0 & 0.0 & 0.0 & 0.01 \\
\hline 19.5 & 0.0 & 0.0 & 0.0 & 0.0 & 0.0 & 0.0 & 0.0 & 0.0 & 0.0 & 0.0 & 0.0 & 0.0 & 0.0 & 0.00 \\
\hline 20.0 & 0.0 & 0.0 & 0.0 & 0.0 & 0.0 & 0.0 & 0.0 & 0.0 & 0.0 & 0.0 & 0.0 & 0.0 & 0.0 & 0.00 \\
\hline
\end{tabular}

Notes. The relative disc-mass loss considering particles bound tighter to the central star than to the encounter for all simulated configurations of prograde parabolic $(e=1)$ star-disc encounters. The first row contains the perturber masses $M_{2}$ in units of $M_{1}$, the first column contains the relative periastra $r_{\text {peri }} / r_{\text {disc }}$. 
M. Steinhausen et al.: Disc-mass distribution in star-disc encounters

Table A.3. Relative disc-mass loss assuming an initial disc-mass distribution of $p=1$.

\begin{tabular}{|c|c|c|c|c|c|c|c|c|c|c|c|c|c|c|}
\hline & $0.1 M_{1}$ & $0.3 M_{1}$ & $0.5 M_{1}$ & $1 M_{1}$ & $1.5 M_{1}$ & $2 M_{1}$ & $3 M_{1}$ & $4 M_{1}$ & $5 M_{1}$ & $9 M_{1}$ & $20 M_{1}$ & $50 M_{1}$ & $90 M_{1}$ & $500 M_{1}$ \\
\hline 00.1 & 0.16 & 0.42 & 0.59 & 0.63 & 0.67 & 0.68 & 0.71 & 0.74 & 0.79 & 0.87 & 0.90 & 0.87 & 0.89 & 0.92 \\
\hline 00.2 & 0.16 & 0.39 & 0.56 & 0.66 & 0.78 & 0.83 & 0.86 & 0.87 & 0.89 & 0.92 & 0.93 & 0.92 & 0.94 & 0.95 \\
\hline 00.3 & 0.15 & 0.35 & 0.53 & 0.70 & 0.78 & 0.83 & 0.87 & 0.91 & 0.93 & 0.95 & 0.96 & 0.98 & 0.99 & 0.98 \\
\hline 00.4 & 0.14 & 0.29 & 0.49 & 0.66 & 0.77 & 0.82 & 0.88 & 0.92 & 0.92 & 0.96 & 0.98 & 0.99 & 0.99 & 0.99 \\
\hline 00.5 & 0.14 & 0.25 & 0.47 & 0.65 & 0.74 & 0.79 & 0.84 & 0.87 & 0.90 & 0.94 & 0.98 & 0.99 & 0.99 & 0.99 \\
\hline 00.6 & 0.13 & 0.23 & 0.45 & 0.63 & 0.69 & 0.74 & 0.80 & 0.83 & 0.86 & 0.92 & 0.97 & 0.98 & 0.99 & 0.99 \\
\hline 00.7 & 0.12 & 0.22 & 0.43 & 0.59 & 0.65 & 0.69 & 0.74 & 0.79 & 0.81 & 0.87 & 0.94 & 0.98 & 0.99 & 0.99 \\
\hline 00.8 & 0.11 & 0.21 & 0.38 & 0.53 & 0.61 & 0.64 & 0.71 & 0.74 & 0.77 & 0.85 & 0.91 & 0.97 & 0.99 & 0.99 \\
\hline 00.9 & 0.10 & 0.19 & 0.34 & 0.49 & 0.55 & 0.60 & 0.66 & 0.70 & 0.73 & 0.81 & 0.89 & 0.95 & 0.97 & 0.99 \\
\hline 01.0 & 0.09 & 0.18 & 0.29 & 0.43 & 0.51 & 0.55 & 0.62 & 0.66 & 0.69 & 0.78 & 0.86 & 0.93 & 0.97 & 0.99 \\
\hline 01.5 & 0.03 & 0.09 & 0.12 & 0.21 & 0.30 & 0.35 & 0.42 & 0.48 & 0.52 & 0.62 & 0.74 & 0.84 & 0.89 & 0.98 \\
\hline 02.0 & 0.00 & 0.01 & 0.04 & 0.09 & 0.13 & 0.18 & 0.25 & 0.31 & 0.36 & 0.47 & 0.62 & 0.75 & 0.82 & 0.95 \\
\hline 02.5 & 0.00 & 0.00 & 0.00 & 0.02 & 0.05 & 0.09 & 0.14 & 0.18 & 0.22 & 0.34 & 0.50 & 0.66 & 0.75 & 0.90 \\
\hline 03.0 & 0.00 & 0.00 & 0.00 & 0.00 & 0.01 & 0.02 & 0.05 & 0.09 & 0.12 & 0.23 & 0.39 & 0.58 & 0.68 & 0.87 \\
\hline 03.5 & 0.00 & 0.00 & 0.00 & 0.00 & 0.00 & 0.00 & 0.02 & 0.04 & 0.06 & 0.14 & 0.29 & 0.49 & 0.60 & 0.82 \\
\hline 04.0 & 0.00 & 0.00 & 0.00 & 0.00 & 0.00 & 0.00 & 0.00 & 0.00 & 0.01 & 0.08 & 0.21 & 0.41 & 0.53 & 0.78 \\
\hline 04.5 & 0.00 & 0.00 & 0.00 & 0.00 & 0.00 & 0.00 & 0.00 & 0.00 & 0.00 & 0.03 & 0.14 & 0.32 & 0.45 & 0.75 \\
\hline 05.0 & 0.0 & 0.00 & 0.0 & 0.00 & 0.00 & 0.00 & 0.00 & 0.00 & 0.00 & 0.01 & 0.10 & 0.26 & 0.40 & 0.70 \\
\hline 05.5 & 0.0 & 0.0 & 0.0 & 0.0 & 0.00 & 0.00 & 0.00 & 0.00 & 0.00 & 0.00 & 0.04 & 0.20 & 0.32 & 0.66 \\
\hline 06.0 & 0.0 & 0.0 & 0.0 & 0.0 & 0.00 & 0.00 & 0.00 & 0.00 & 0.00 & 0.00 & 0.03 & 0.16 & 0.26 & 0.62 \\
\hline 06.5 & 0.0 & 0.0 & 0.0 & 0.0 & 0.0 & 0.00 & 0.00 & 0.00 & 0.00 & 0.00 & 0.01 & 0.10 & 0.22 & 0.58 \\
\hline 07.0 & 0.0 & 0.0 & 0.0 & 0.0 & 0.0 & 0.00 & 0.00 & 0.00 & 0.00 & 0.00 & 0.00 & 0.09 & 0.16 & 0.53 \\
\hline 07.5 & 0.0 & 0.0 & 0.0 & 0.0 & 0.0 & 0.0 & 0.0 & 0.0 & 0.00 & 0.00 & 0.00 & 0.04 & 0.14 & 0.50 \\
\hline 08.0 & 0.0 & 0.0 & 0.0 & 0.0 & 0.0 & 0.0 & 0.0 & 0.0 & 0.00 & 0.00 & 0.00 & 0.03 & 0.12 & 0.46 \\
\hline 08.5 & 0.0 & 0.0 & 0.0 & 0.0 & 0.0 & 0.0 & 0.0 & 0.0 & 0.0 & 0.00 & 0.00 & 0.01 & 0.08 & 0.43 \\
\hline 09.0 & 0.0 & 0.0 & 0.0 & 0.0 & 0.0 & 0.0 & 0.0 & 0.0 & 0.0 & 0.00 & 0.00 & 0.00 & 0.07 & 0.38 \\
\hline 09.5 & 0.0 & 0.0 & 0.0 & 0.0 & 0.0 & 0.0 & 0.0 & 0.0 & 0.0 & 0.0 & 0.00 & 0.00 & 0.03 & 0.35 \\
\hline 10.0 & 0.0 & 0.0 & 0.0 & 0.0 & 0.0 & 0.0 & 0.0 & 0.0 & 0.0 & 0.0 & 0.00 & 0.00 & 0.03 & 0.30 \\
\hline 10.5 & 0.0 & 0.0 & 0.0 & 0.0 & 0.0 & 0.0 & 0.0 & 0.0 & 0.0 & 0.0 & 0.0 & 0.00 & 0.01 & 0.27 \\
\hline 11.0 & 0.0 & 0.0 & 0.0 & 0.0 & 0.0 & 0.0 & 0.0 & 0.0 & 0.0 & 0.0 & 0.0 & 0.00 & 0.00 & 0.24 \\
\hline 11.5 & 0.0 & 0.0 & 0.0 & 0.0 & 0.0 & 0.0 & 0.0 & 0.0 & 0.0 & 0.0 & 0.0 & 0.00 & 0.00 & 0.22 \\
\hline 12.0 & 0.0 & 0.0 & 0.0 & 0.0 & 0.0 & 0.0 & 0.0 & 0.0 & 0.0 & 0.0 & 0.0 & 0.00 & 0.00 & 0.19 \\
\hline 12.5 & 0.0 & 0.0 & 0.0 & 0.0 & 0.0 & 0.0 & 0.0 & 0.0 & 0.0 & 0.0 & 0.0 & 0.00 & 0.00 & 0.17 \\
\hline 13.0 & 0.0 & 0.0 & 0.0 & 0.0 & 0.0 & 0.0 & 0.0 & 0.0 & 0.0 & 0.0 & 0.0 & 0.00 & 0.00 & 0.14 \\
\hline 13.5 & 0.0 & 0.0 & 0.0 & 0.0 & 0.0 & 0.0 & 0.0 & 0.0 & 0.0 & 0.0 & 0.0 & 0.00 & 0.00 & 0.14 \\
\hline 14.0 & 0.0 & 0.0 & 0.0 & 0.0 & 0.0 & 0.0 & 0.0 & 0.0 & 0.0 & 0.0 & 0.0 & 0.00 & 0.00 & 0.10 \\
\hline 14.5 & 0.0 & 0.0 & 0.0 & 0.0 & 0.0 & 0.0 & 0.0 & 0.0 & 0.0 & 0.0 & 0.0 & 0.00 & 0.00 & 0.10 \\
\hline 15.0 & 0.0 & 0.0 & 0.0 & 0.0 & 0.0 & 0.0 & 0.0 & 0.0 & 0.0 & 0.0 & 0.0 & 0.00 & 0.00 & 0.07 \\
\hline 15.5 & 0.0 & 0.0 & 0.0 & 0.0 & 0.0 & 0.0 & 0.0 & 0.0 & 0.0 & 0.0 & 0.0 & 0.0 & 0.00 & 0.07 \\
\hline 16.0 & 0.0 & 0.0 & 0.0 & 0.0 & 0.0 & 0.0 & 0.0 & 0.0 & 0.0 & 0.0 & 0.0 & 0.0 & 0.00 & 0.07 \\
\hline 16.5 & 0.0 & 0.0 & 0.0 & 0.0 & 0.0 & 0.0 & 0.0 & 0.0 & 0.0 & 0.0 & 0.0 & 0.0 & 0.00 & 0.05 \\
\hline 17.0 & 0.0 & 0.0 & 0.0 & 0.0 & 0.0 & 0.0 & 0.0 & 0.0 & 0.0 & 0.0 & 0.0 & 0.0 & 0.00 & 0.04 \\
\hline 17.5 & 0.0 & 0.0 & 0.0 & 0.0 & 0.0 & 0.0 & 0.0 & 0.0 & 0.0 & 0.0 & 0.0 & 0.0 & 0.0 & 0.02 \\
\hline 18.0 & 0.0 & 0.0 & 0.0 & 0.0 & 0.0 & 0.0 & 0.0 & 0.0 & 0.0 & 0.0 & 0.0 & 0.0 & 0.0 & 0.02 \\
\hline 18.5 & 0.0 & 0.0 & 0.0 & 0.0 & 0.0 & 0.0 & 0.0 & 0.0 & 0.0 & 0.0 & 0.0 & 0.0 & 0.0 & 0.01 \\
\hline 19.0 & 0.0 & 0.0 & 0.0 & 0.0 & 0.0 & 0.0 & 0.0 & 0.0 & 0.0 & 0.0 & 0.0 & 0.0 & 0.0 & 0.01 \\
\hline 19.5 & 0.0 & 0.0 & 0.0 & 0.0 & 0.0 & 0.0 & 0.0 & 0.0 & 0.0 & 0.0 & 0.0 & 0.0 & 0.0 & 0.00 \\
\hline 20.0 & 0.0 & 0.0 & 0.0 & 0.0 & 0.0 & 0.0 & 0.0 & 0.0 & 0.0 & 0.0 & 0.0 & 0.0 & 0.0 & 0.00 \\
\hline
\end{tabular}

Notes. The relative disc-mass loss considering particles bound tighter to the central star than to the encounter for all simulated configurations of prograde parabolic $(e=1)$ star-disc encounters. The first row contains the perturber masses $M_{2}$ in units of $M_{1}$, the first column contains the relative periastra $r_{\text {peri }} / r_{\text {disc }}$. 
Table A.4. Relative disc-mass loss assuming an initial disc-mass distribution of $p=7 / 4$.

\begin{tabular}{|c|c|c|c|c|c|c|c|c|c|c|c|c|c|c|}
\hline & $0.1 M_{1}$ & $0.3 M_{1}$ & $0.5 M_{1}$ & $1 M_{1}$ & $1.5 M_{1}$ & $2 M_{1}$ & $3 M_{1}$ & $4 M_{1}$ & $5 M_{1}$ & $9 M_{1}$ & $20 M_{1}$ & $50 M_{1}$ & $90 M_{1}$ & $500 M_{1}$ \\
\hline 00.1 & 0.16 & 0.41 & 0.59 & 0.67 & 0.74 & 0.75 & 0.78 & 0.80 & 0.84 & 0.90 & 0.92 & 0.90 & 0.92 & 0.94 \\
\hline 00.2 & 0.15 & 0.37 & 0.55 & 0.69 & 0.80 & 0.85 & 0.89 & 0.91 & 0.92 & 0.94 & 0.95 & 0.95 & 0.96 & 0.97 \\
\hline 00.3 & 0.14 & 0.30 & 0.49 & 0.66 & 0.74 & 0.81 & 0.86 & 0.90 & 0.93 & 0.96 & 0.97 & 0.99 & 0.99 & 0.99 \\
\hline 00.4 & 0.12 & 0.24 & 0.42 & 0.58 & 0.68 & 0.73 & 0.81 & 0.86 & 0.87 & 0.94 & 0.98 & 0.99 & 0.99 & 0.99 \\
\hline 00.5 & 0.11 & 0.21 & 0.37 & 0.53 & 0.61 & 0.66 & 0.72 & 0.77 & 0.82 & 0.88 & 0.96 & 0.99 & 0.99 & 0.99 \\
\hline 00.6 & 0.10 & 0.18 & 0.34 & 0.48 & 0.54 & 0.59 & 0.66 & 0.70 & 0.74 & 0.83 & 0.94 & 0.98 & 0.99 & 0.99 \\
\hline 00.7 & 0.08 & 0.16 & 0.31 & 0.43 & 0.49 & 0.53 & 0.59 & 0.64 & 0.67 & 0.75 & 0.87 & 0.97 & 0.99 & 0.99 \\
\hline 00.8 & 0.08 & 0.15 & 0.26 & 0.38 & 0.44 & 0.48 & 0.54 & 0.58 & 0.61 & 0.71 & 0.82 & 0.94 & 0.98 & 0.99 \\
\hline 00.9 & 0.07 & 0.13 & 0.22 & 0.33 & 0.39 & 0.44 & 0.49 & 0.53 & 0.57 & 0.66 & 0.78 & 0.90 & 0.95 & 0.99 \\
\hline 01.0 & 0.06 & 0.11 & 0.19 & 0.29 & 0.35 & 0.39 & 0.45 & 0.49 & 0.52 & 0.61 & 0.73 & 0.85 & 0.94 & 0.99 \\
\hline 01.5 & 0.01 & 0.05 & 0.07 & 0.13 & 0.18 & 0.22 & 0.27 & 0.32 & 0.35 & 0.44 & 0.57 & 0.70 & 0.78 & 0.98 \\
\hline 02.0 & 0.00 & 0.01 & 0.02 & 0.05 & 0.07 & 0.11 & 0.15 & 0.19 & 0.22 & 0.31 & 0.44 & 0.57 & 0.67 & 0.89 \\
\hline 02.5 & 0.00 & 0.00 & 0.00 & 0.01 & 0.03 & 0.05 & 0.08 & 0.10 & 0.13 & 0.21 & 0.33 & 0.47 & 0.57 & 0.79 \\
\hline 03.0 & 0.00 & 0.00 & 0.00 & 0.00 & 0.01 & 0.01 & 0.03 & 0.05 & 0.06 & 0.13 & 0.24 & 0.39 & 0.49 & 0.74 \\
\hline 03.5 & 0.00 & 0.00 & 0.00 & 0.00 & 0.00 & 0.00 & 0.01 & 0.02 & 0.03 & 0.08 & 0.17 & 0.32 & 0.41 & 0.67 \\
\hline 04.0 & 0.00 & 0.00 & 0.00 & 0.00 & 0.00 & 0.00 & 0.00 & 0.00 & 0.01 & 0.05 & 0.12 & 0.25 & 0.35 & 0.61 \\
\hline 04.5 & 0.00 & 0.00 & 0.00 & 0.00 & 0.00 & 0.00 & 0.00 & 0.00 & 0.00 & 0.01 & 0.08 & 0.19 & 0.29 & 0.57 \\
\hline 05.0 & 0.0 & 0.00 & 0.0 & 0.00 & 0.00 & 0.00 & 0.00 & 0.00 & 0.00 & 0.01 & 0.05 & 0.15 & 0.25 & 0.51 \\
\hline 05.5 & 0.0 & 0.0 & 0.0 & 0.0 & 0.00 & 0.00 & 0.00 & 0.00 & 0.00 & 0.00 & 0.02 & 0.11 & 0.19 & 0.47 \\
\hline 06.0 & 0.0 & 0.0 & 0.0 & 0.0 & 0.00 & 0.00 & 0.00 & 0.00 & 0.00 & 0.00 & 0.01 & 0.09 & 0.15 & 0.43 \\
\hline 06.5 & 0.0 & 0.0 & 0.0 & 0.0 & 0.0 & 0.00 & 0.00 & 0.00 & 0.00 & 0.00 & 0.01 & 0.06 & 0.12 & 0.39 \\
\hline 07.0 & 0.0 & 0.0 & 0.0 & 0.0 & 0.0 & 0.00 & 0.00 & 0.00 & 0.00 & 0.00 & 0.00 & 0.05 & 0.09 & 0.35 \\
\hline 07.5 & 0.0 & 0.0 & 0.0 & 0.0 & 0.0 & 0.0 & 0.0 & 0.0 & 0.00 & 0.00 & 0.00 & 0.02 & 0.08 & 0.33 \\
\hline 08.0 & 0.0 & 0.0 & 0.0 & 0.0 & 0.0 & 0.0 & 0.0 & 0.0 & 0.00 & 0.00 & 0.00 & 0.02 & 0.07 & 0.29 \\
\hline 08.5 & 0.0 & 0.0 & 0.0 & 0.0 & 0.0 & 0.0 & 0.0 & 0.0 & 0.0 & 0.00 & 0.00 & 0.00 & 0.04 & 0.27 \\
\hline 09.0 & 0.0 & 0.0 & 0.0 & 0.0 & 0.0 & 0.0 & 0.0 & 0.0 & 0.0 & 0.00 & 0.00 & 0.00 & 0.04 & 0.23 \\
\hline 09.5 & 0.0 & 0.0 & 0.0 & 0.0 & 0.0 & 0.0 & 0.0 & 0.0 & 0.0 & 0.0 & 0.00 & 0.00 & 0.02 & 0.21 \\
\hline 10.0 & 0.0 & 0.0 & 0.0 & 0.0 & 0.0 & 0.0 & 0.0 & 0.0 & 0.0 & 0.0 & 0.00 & 0.00 & 0.01 & 0.17 \\
\hline 10.5 & 0.0 & 0.0 & 0.0 & 0.0 & 0.0 & 0.0 & 0.0 & 0.0 & 0.0 & 0.0 & 0.0 & 0.00 & 0.00 & 0.16 \\
\hline 11.0 & 0.0 & 0.0 & 0.0 & 0.0 & 0.0 & 0.0 & 0.0 & 0.0 & 0.0 & 0.0 & 0.0 & 0.00 & 0.00 & 0.14 \\
\hline 11.5 & 0.0 & 0.0 & 0.0 & 0.0 & 0.0 & 0.0 & 0.0 & 0.0 & 0.0 & 0.0 & 0.0 & 0.00 & 0.00 & 0.12 \\
\hline 12.0 & 0.0 & 0.0 & 0.0 & 0.0 & 0.0 & 0.0 & 0.0 & 0.0 & 0.0 & 0.0 & 0.0 & 0.00 & 0.00 & 0.10 \\
\hline 12.5 & 0.0 & 0.0 & 0.0 & 0.0 & 0.0 & 0.0 & 0.0 & 0.0 & 0.0 & 0.0 & 0.0 & 0.00 & 0.00 & 0.10 \\
\hline 13.0 & 0.0 & 0.0 & 0.0 & 0.0 & 0.0 & 0.0 & 0.0 & 0.0 & 0.0 & 0.0 & 0.0 & 0.00 & 0.00 & 0.08 \\
\hline 13.5 & 0.0 & 0.0 & 0.0 & 0.0 & 0.0 & 0.0 & 0.0 & 0.0 & 0.0 & 0.0 & 0.0 & 0.00 & 0.00 & 0.07 \\
\hline 14.0 & 0.0 & 0.0 & 0.0 & 0.0 & 0.0 & 0.0 & 0.0 & 0.0 & 0.0 & 0.0 & 0.0 & 0.00 & 0.00 & 0.06 \\
\hline 14.5 & 0.0 & 0.0 & 0.0 & 0.0 & 0.0 & 0.0 & 0.0 & 0.0 & 0.0 & 0.0 & 0.0 & 0.00 & 0.00 & 0.05 \\
\hline 15.0 & 0.0 & 0.0 & 0.0 & 0.0 & 0.0 & 0.0 & 0.0 & 0.0 & 0.0 & 0.0 & 0.0 & 0.00 & 0.00 & 0.04 \\
\hline 15.5 & 0.0 & 0.0 & 0.0 & 0.0 & 0.0 & 0.0 & 0.0 & 0.0 & 0.0 & 0.0 & 0.0 & 0.0 & 0.00 & 0.04 \\
\hline 16.0 & 0.0 & 0.0 & 0.0 & 0.0 & 0.0 & 0.0 & 0.0 & 0.0 & 0.0 & 0.0 & 0.0 & 0.0 & 0.00 & 0.04 \\
\hline 16.5 & 0.0 & 0.0 & 0.0 & 0.0 & 0.0 & 0.0 & 0.0 & 0.0 & 0.0 & 0.0 & 0.0 & 0.0 & 0.00 & 0.02 \\
\hline 17.0 & 0.0 & 0.0 & 0.0 & 0.0 & 0.0 & 0.0 & 0.0 & 0.0 & 0.0 & 0.0 & 0.0 & 0.0 & 0.00 & 0.02 \\
\hline 17.5 & 0.0 & 0.0 & 0.0 & 0.0 & 0.0 & 0.0 & 0.0 & 0.0 & 0.0 & 0.0 & 0.0 & 0.0 & 0.0 & 0.01 \\
\hline 18.0 & 0.0 & 0.0 & 0.0 & 0.0 & 0.0 & 0.0 & 0.0 & 0.0 & 0.0 & 0.0 & 0.0 & 0.0 & 0.0 & 0.01 \\
\hline 18.5 & 0.0 & 0.0 & 0.0 & 0.0 & 0.0 & 0.0 & 0.0 & 0.0 & 0.0 & 0.0 & 0.0 & 0.0 & 0.0 & 0.00 \\
\hline 19.0 & 0.0 & 0.0 & 0.0 & 0.0 & 0.0 & 0.0 & 0.0 & 0.0 & 0.0 & 0.0 & 0.0 & 0.0 & 0.0 & 0.00 \\
\hline 19.5 & 0.0 & 0.0 & 0.0 & 0.0 & 0.0 & 0.0 & 0.0 & 0.0 & 0.0 & 0.0 & 0.0 & 0.0 & 0.0 & 0.00 \\
\hline 20.0 & 0.0 & 0.0 & 0.0 & 0.0 & 0.0 & 0.0 & 0.0 & 0.0 & 0.0 & 0.0 & 0.0 & 0.0 & 0.0 & 0.00 \\
\hline
\end{tabular}

Notes. The relative disc-mass loss considering particles bound tighter to the central star than to the encounter for all simulated configurations of prograde parabolic $(e=1)$ star-disc encounters. The first row contains the perturber masses $M_{2}$ in units of $M_{1}$, the first column contains the relative periastra $r_{\text {peri }} / r_{\text {disc }}$. 
M. Steinhausen et al.: Disc-mass distribution in star-disc encounters

Appendix B: Relative angular momentum loss

Table B.1. Relative angular momentum loss assuming an initial disc-mass distribution of $p=0$.

\begin{tabular}{|c|c|c|c|c|c|c|c|c|c|c|c|c|c|c|}
\hline & $0.1 M_{1}$ & $0.3 M_{1}$ & $0.5 M_{1}$ & $1 M_{1}$ & $1.5 M_{1}$ & $2 M_{1}$ & $3 M_{1}$ & $4 M_{1}$ & $5 M_{1}$ & $\overline{9 M_{1}}$ & $20 M_{1}$ & $\overline{50 M_{1}}$ & $\overline{90 M_{1}}$ & $\overline{500 M_{1}}$ \\
\hline 00.1 & 0.25 & 0.52 & 0.64 & 0.68 & 0.67 & 0.68 & 0.72 & 0.68 & 0.77 & 0.87 & 0.91 & 0.93 & 0.90 & 0.94 \\
\hline 00.2 & 0.22 & 0.43 & 0.59 & 0.68 & 0.80 & 0.85 & 0.84 & 0.83 & 0.87 & 0.93 & 0.94 & 0.93 & 0.93 & 0.96 \\
\hline 00.3 & 0.20 & 0.36 & 0.60 & 0.80 & 0.85 & 0.87 & 0.90 & 0.92 & 0.94 & 0.96 & 0.96 & 0.99 & 0.99 & 0.99 \\
\hline 00.4 & 0.18 & 0.25 & 0.61 & 0.80 & 0.87 & 0.91 & 0.95 & 0.97 & 0.97 & 0.98 & 0.98 & 0.99 & 0.99 & 0.99 \\
\hline 00.5 & 0.17 & 0.19 & 0.59 & 0.82 & 0.90 & 0.93 & 0.95 & 0.97 & 0.98 & 0.98 & 0.99 & 1.00 & 0.99 & 1.00 \\
\hline 00.6 & 0.17 & 0.17 & 0.59 & 0.85 & 0.90 & 0.92 & 0.94 & 0.95 & 0.97 & 0.99 & 0.99 & 1.00 & 1.00 & 1.00 \\
\hline 00.7 & 0.17 & 0.16 & 0.63 & 0.82 & 0.88 & 0.90 & 0.92 & 0.95 & 0.96 & 0.98 & 0.99 & 1.00 & 1.00 & 1.00 \\
\hline 00.8 & 0.16 & 0.20 & 0.59 & 0.79 & 0.85 & 0.88 & 0.91 & 0.93 & 0.94 & 0.97 & 0.99 & 0.99 & 1.00 & 1.00 \\
\hline 00.9 & 0.16 & 0.23 & 0.54 & 0.75 & 0.81 & 0.85 & 0.89 & 0.91 & 0.93 & 0.96 & 0.98 & 0.99 & 0.99 & 1.00 \\
\hline 01.0 & 0.16 & 0.26 & 0.49 & 0.70 & 0.78 & 0.82 & 0.87 & 0.89 & 0.91 & 0.95 & 0.98 & 0.99 & 0.99 & 1.00 \\
\hline 01.5 & 0.07 & 0.22 & 0.27 & 0.46 & 0.58 & 0.64 & 0.73 & 0.78 & 0.81 & 0.88 & 0.94 & 0.97 & 0.98 & 1.00 \\
\hline 02.0 & 0.00 & 0.07 & 0.15 & 0.25 & 0.34 & 0.44 & 0.55 & 0.62 & 0.67 & 0.77 & 0.88 & 0.95 & 0.97 & 0.99 \\
\hline 02.5 & 0.00 & 0.01 & 0.03 & 0.12 & 0.20 & 0.26 & 0.37 & 0.45 & 0.51 & 0.66 & 0.80 & 0.91 & 0.94 & 0.98 \\
\hline 03.0 & 0.00 & 0.00 & 0.00 & 0.03 & 0.09 & 0.13 & 0.21 & 0.28 & 0.34 & 0.53 & 0.71 & 0.86 & 0.92 & 0.98 \\
\hline 03.5 & 0.00 & 0.00 & 0.00 & 0.01 & 0.02 & 0.05 & 0.12 & 0.17 & 0.22 & 0.38 & 0.61 & 0.80 & 0.88 & 0.97 \\
\hline 04.0 & 0.00 & 0.00 & 0.00 & 0.00 & 0.01 & 0.01 & 0.04 & 0.07 & 0.12 & 0.28 & 0.51 & 0.73 & 0.83 & 0.96 \\
\hline 04.5 & 0.00 & 0.00 & 0.00 & 0.00 & 0.00 & 0.00 & 0.01 & 0.03 & 0.05 & 0.16 & 0.40 & 0.65 & 0.78 & 0.94 \\
\hline 05.0 & 0.0 & 0.00 & 0.0 & 0.00 & 0.00 & 0.00 & 0.00 & 0.01 & 0.02 & 0.10 & 0.32 & 0.58 & 0.73 & 0.93 \\
\hline 05.5 & 0.0 & 0.0 & 0.0 & 0.0 & 0.00 & 0.00 & 0.00 & 0.00 & 0.01 & 0.04 & 0.21 & 0.50 & 0.65 & 0.91 \\
\hline 06.0 & 0.0 & 0.0 & 0.0 & 0.0 & 0.00 & 0.00 & 0.00 & 0.00 & 0.00 & 0.02 & 0.16 & 0.43 & 0.59 & 0.89 \\
\hline 06.5 & 0.0 & 0.0 & 0.0 & 0.0 & 0.0 & 0.00 & 0.00 & 0.00 & 0.00 & 0.01 & 0.11 & 0.34 & 0.52 & 0.87 \\
\hline 07.0 & 0.0 & 0.0 & 0.0 & 0.0 & 0.0 & 0.00 & 0.00 & 0.00 & 0.00 & 0.00 & 0.05 & 0.30 & 0.45 & 0.84 \\
\hline 07.5 & 0.0 & 0.0 & 0.0 & 0.0 & 0.0 & 0.0 & 0.0 & 0.0 & 0.00 & 0.00 & 0.04 & 0.21 & 0.40 & 0.81 \\
\hline 08.0 & 0.0 & 0.0 & 0.0 & 0.0 & 0.0 & 0.0 & 0.0 & 0.0 & 0.00 & 0.00 & 0.01 & 0.17 & 0.36 & 0.78 \\
\hline 08.5 & 0.0 & 0.0 & 0.0 & 0.0 & 0.0 & 0.0 & 0.0 & 0.0 & 0.0 & 0.00 & 0.01 & 0.11 & 0.29 & 0.75 \\
\hline 09.0 & 0.0 & 0.0 & 0.0 & 0.0 & 0.0 & 0.0 & 0.0 & 0.0 & 0.0 & 0.00 & 0.00 & 0.08 & 0.25 & 0.71 \\
\hline 09.5 & 0.0 & 0.0 & 0.0 & 0.0 & 0.0 & 0.0 & 0.0 & 0.0 & 0.0 & 0.0 & 0.00 & 0.06 & 0.18 & 0.68 \\
\hline 10.0 & 0.0 & 0.0 & 0.0 & 0.0 & 0.0 & 0.0 & 0.0 & 0.0 & 0.0 & 0.0 & 0.00 & 0.03 & 0.16 & 0.63 \\
\hline 10.5 & 0.0 & 0.0 & 0.0 & 0.0 & 0.0 & 0.0 & 0.0 & 0.0 & 0.0 & 0.0 & 0.0 & 0.03 & 0.10 & 0.60 \\
\hline 11.0 & 0.0 & 0.0 & 0.0 & 0.0 & 0.0 & 0.0 & 0.0 & 0.0 & 0.0 & 0.0 & 0.0 & 0.01 & 0.08 & 0.56 \\
\hline 11.5 & 0.0 & 0.0 & 0.0 & 0.0 & 0.0 & 0.0 & 0.0 & 0.0 & 0.0 & 0.0 & 0.0 & 0.01 & 0.05 & 0.52 \\
\hline 12.0 & 0.0 & 0.0 & 0.0 & 0.0 & 0.0 & 0.0 & 0.0 & 0.0 & 0.0 & 0.0 & 0.0 & 0.00 & 0.04 & 0.48 \\
\hline 12.5 & 0.0 & 0.0 & 0.0 & 0.0 & 0.0 & 0.0 & 0.0 & 0.0 & 0.0 & 0.0 & 0.0 & 0.00 & 0.02 & 0.45 \\
\hline 13.0 & 0.0 & 0.0 & 0.0 & 0.0 & 0.0 & 0.0 & 0.0 & 0.0 & 0.0 & 0.0 & 0.0 & 0.00 & 0.02 & 0.41 \\
\hline 13.5 & 0.0 & 0.0 & 0.0 & 0.0 & 0.0 & 0.0 & 0.0 & 0.0 & 0.0 & 0.0 & 0.0 & 0.00 & 0.01 & 0.39 \\
\hline 14.0 & 0.0 & 0.0 & 0.0 & 0.0 & 0.0 & 0.0 & 0.0 & 0.0 & 0.0 & 0.0 & 0.0 & 0.00 & 0.01 & 0.34 \\
\hline 14.5 & 0.0 & 0.0 & 0.0 & 0.0 & 0.0 & 0.0 & 0.0 & 0.0 & 0.0 & 0.0 & 0.0 & 0.00 & 0.01 & 0.33 \\
\hline 15.0 & 0.0 & 0.0 & 0.0 & 0.0 & 0.0 & 0.0 & 0.0 & 0.0 & 0.0 & 0.0 & 0.0 & 0.00 & 0.00 & 0.28 \\
\hline 15.5 & 0.0 & 0.0 & 0.0 & 0.0 & 0.0 & 0.0 & 0.0 & 0.0 & 0.0 & 0.0 & 0.0 & 0.0 & 0.00 & 0.26 \\
\hline 16.0 & 0.0 & 0.0 & 0.0 & 0.0 & 0.0 & 0.0 & 0.0 & 0.0 & 0.0 & 0.0 & 0.0 & 0.0 & 0.00 & 0.25 \\
\hline 16.5 & 0.0 & 0.0 & 0.0 & 0.0 & 0.0 & 0.0 & 0.0 & 0.0 & 0.0 & 0.0 & 0.0 & 0.0 & 0.00 & 0.21 \\
\hline 17.0 & 0.0 & 0.0 & 0.0 & 0.0 & 0.0 & 0.0 & 0.0 & 0.0 & 0.0 & 0.0 & 0.0 & 0.0 & 0.00 & 0.20 \\
\hline 17.5 & 0.0 & 0.0 & 0.0 & 0.0 & 0.0 & 0.0 & 0.0 & 0.0 & 0.0 & 0.0 & 0.0 & 0.0 & 0.0 & 0.15 \\
\hline 18.0 & 0.0 & 0.0 & 0.0 & 0.0 & 0.0 & 0.0 & 0.0 & 0.0 & 0.0 & 0.0 & 0.0 & 0.0 & 0.0 & 0.15 \\
\hline 18.5 & 0.0 & 0.0 & 0.0 & 0.0 & 0.0 & 0.0 & 0.0 & 0.0 & 0.0 & 0.0 & 0.0 & 0.0 & 0.0 & 0.10 \\
\hline 19.0 & 0.0 & 0.0 & 0.0 & 0.0 & 0.0 & 0.0 & 0.0 & 0.0 & 0.0 & 0.0 & 0.0 & 0.0 & 0.0 & 0.10 \\
\hline 19.5 & 0.0 & 0.0 & 0.0 & 0.0 & 0.0 & 0.0 & 0.0 & 0.0 & 0.0 & 0.0 & 0.0 & 0.0 & 0.0 & 0.07 \\
\hline 20.0 & 0.0 & 0.0 & 0.0 & 0.0 & 0.0 & 0.0 & 0.0 & 0.0 & 0.0 & 0.0 & 0.0 & 0.0 & 0.0 & 0.07 \\
\hline
\end{tabular}

Notes. The relative angular momentum loss considering particles bound tighter to the central star than to the encounter for all simulated configurations of prograde parabolic $(e=1)$ star-disc encounters. The first row contains the perturber masses $M_{2}$ in units of $M_{1}$, the first column contains the relative periastra $r_{\text {peri }} / r_{\text {disc }}$. 
Table B.2. Relative angular momentum loss assuming an initial disc-mass distribution of $p=1 / 2$.

\begin{tabular}{|c|c|c|c|c|c|c|c|c|c|c|c|c|c|c|}
\hline & $0.1 M_{1}$ & $0.3 M_{1}$ & $0.5 M_{1}$ & $1 M_{1}$ & $1.5 M_{1}$ & $2 M_{1}$ & $3 M_{1}$ & $4 M_{1}$ & $5 M_{1}$ & $9 M_{1}$ & $20 M_{1}$ & $50 M_{1}$ & $90 M_{1}$ & $500 M_{1}$ \\
\hline 00.1 & 0.24 & 0.51 & 0.63 & 0.68 & 0.68 & 0.70 & 0.73 & 0.69 & 0.78 & 0.87 & 0.92 & 0.93 & 0.90 & 0.94 \\
\hline 00.2 & 0.22 & 0.42 & 0.59 & 0.70 & 0.81 & 0.85 & 0.85 & 0.85 & 0.88 & 0.94 & 0.95 & 0.94 & 0.93 & 0.96 \\
\hline 00.3 & 0.19 & 0.34 & 0.60 & 0.80 & 0.85 & 0.88 & 0.90 & 0.93 & 0.94 & 0.96 & 0.96 & 0.99 & 0.99 & 0.99 \\
\hline 00.4 & 0.18 & 0.25 & 0.60 & 0.79 & 0.87 & 0.90 & 0.94 & 0.97 & 0.97 & 0.98 & 0.99 & 0.99 & 0.99 & 0.99 \\
\hline 00.5 & 0.17 & 0.19 & 0.58 & 0.81 & 0.89 & 0.91 & 0.94 & 0.95 & 0.97 & 0.98 & 0.99 & 1.00 & 0.99 & 1.00 \\
\hline 00.6 & 0.16 & 0.17 & 0.57 & 0.82 & 0.87 & 0.89 & 0.93 & 0.94 & 0.95 & 0.98 & 0.99 & 1.00 & 1.00 & 1.00 \\
\hline 00.7 & 0.16 & 0.16 & 0.59 & 0.78 & 0.84 & 0.87 & 0.90 & 0.92 & 0.94 & 0.96 & 0.98 & 0.99 & 1.00 & 1.00 \\
\hline 00.8 & 0.15 & 0.20 & 0.55 & 0.75 & 0.81 & 0.84 & 0.88 & 0.90 & 0.92 & 0.95 & 0.98 & 0.99 & 1.00 & 1.00 \\
\hline 00.9 & 0.15 & 0.22 & 0.50 & 0.70 & 0.77 & 0.81 & 0.85 & 0.88 & 0.90 & 0.94 & 0.97 & 0.99 & 0.99 & 1.00 \\
\hline 01.0 & 0.15 & 0.25 & 0.44 & 0.65 & 0.73 & 0.77 & 0.83 & 0.86 & 0.88 & 0.93 & 0.96 & 0.98 & 0.99 & 1.00 \\
\hline 01.5 & 0.06 & 0.19 & 0.24 & 0.41 & 0.52 & 0.58 & 0.67 & 0.72 & 0.76 & 0.83 & 0.91 & 0.95 & 0.97 & 0.99 \\
\hline 02.0 & 0.00 & 0.06 & 0.12 & 0.22 & 0.30 & 0.39 & 0.49 & 0.56 & 0.60 & 0.72 & 0.84 & 0.92 & 0.95 & 0.99 \\
\hline 02.5 & 0.00 & 0.01 & 0.03 & 0.10 & 0.17 & 0.23 & 0.32 & 0.39 & 0.45 & 0.60 & 0.75 & 0.86 & 0.91 & 0.97 \\
\hline 03.0 & 0.00 & 0.00 & 0.00 & 0.03 & 0.07 & 0.11 & 0.18 & 0.24 & 0.30 & 0.46 & 0.64 & 0.81 & 0.88 & 0.96 \\
\hline 03.5 & 0.00 & 0.00 & 0.00 & 0.01 & 0.02 & 0.04 & 0.10 & 0.14 & 0.19 & 0.33 & 0.55 & 0.74 & 0.83 & 0.95 \\
\hline 04.0 & 0.00 & 0.00 & 0.00 & 0.00 & 0.00 & 0.01 & 0.03 & 0.06 & 0.10 & 0.24 & 0.45 & 0.67 & 0.78 & 0.93 \\
\hline 04.5 & 0.00 & 0.00 & 0.00 & 0.00 & 0.00 & 0.00 & 0.01 & 0.03 & 0.05 & 0.14 & 0.34 & 0.59 & 0.71 & 0.91 \\
\hline 05.0 & 0.0 & 0.00 & 0.0 & 0.00 & 0.00 & 0.00 & 0.00 & 0.01 & 0.02 & 0.09 & 0.27 & 0.51 & 0.66 & 0.89 \\
\hline 05.5 & 0.0 & 0.0 & 0.0 & 0.0 & 0.00 & 0.00 & 0.00 & 0.00 & 0.01 & 0.04 & 0.18 & 0.43 & 0.58 & 0.87 \\
\hline 06.0 & 0.0 & 0.0 & 0.0 & 0.0 & 0.00 & 0.00 & 0.00 & 0.00 & 0.00 & 0.02 & 0.13 & 0.37 & 0.52 & 0.85 \\
\hline 06.5 & 0.0 & 0.0 & 0.0 & 0.0 & 0.0 & 0.00 & 0.00 & 0.00 & 0.00 & 0.01 & 0.09 & 0.29 & 0.46 & 0.81 \\
\hline 07.0 & 0.0 & 0.0 & 0.0 & 0.0 & 0.0 & 0.00 & 0.00 & 0.00 & 0.00 & 0.00 & 0.05 & 0.25 & 0.39 & 0.78 \\
\hline 07.5 & 0.0 & 0.0 & 0.0 & 0.0 & 0.0 & 0.0 & 0.0 & 0.0 & 0.00 & 0.00 & 0.03 & 0.18 & 0.34 & 0.75 \\
\hline 08.0 & 0.0 & 0.0 & 0.0 & 0.0 & 0.0 & 0.0 & 0.0 & 0.0 & 0.00 & 0.00 & 0.01 & 0.15 & 0.31 & 0.72 \\
\hline 08.5 & 0.0 & 0.0 & 0.0 & 0.0 & 0.0 & 0.0 & 0.0 & 0.0 & 0.0 & 0.00 & 0.01 & 0.09 & 0.24 & 0.69 \\
\hline 09.0 & 0.0 & 0.0 & 0.0 & 0.0 & 0.0 & 0.0 & 0.0 & 0.0 & 0.0 & 0.00 & 0.00 & 0.07 & 0.22 & 0.65 \\
\hline 09.5 & 0.0 & 0.0 & 0.0 & 0.0 & 0.0 & 0.0 & 0.0 & 0.0 & 0.0 & 0.0 & 0.00 & 0.05 & 0.16 & 0.62 \\
\hline 10.0 & 0.0 & 0.0 & 0.0 & 0.0 & 0.0 & 0.0 & 0.0 & 0.0 & 0.0 & 0.0 & 0.00 & 0.03 & 0.13 & 0.57 \\
\hline 10.5 & 0.0 & 0.0 & 0.0 & 0.0 & 0.0 & 0.0 & 0.0 & 0.0 & 0.0 & 0.0 & 0.0 & 0.02 & 0.09 & 0.53 \\
\hline 11.0 & 0.0 & 0.0 & 0.0 & 0.0 & 0.0 & 0.0 & 0.0 & 0.0 & 0.0 & 0.0 & 0.0 & 0.01 & 0.07 & 0.49 \\
\hline 11.5 & 0.0 & 0.0 & 0.0 & 0.0 & 0.0 & 0.0 & 0.0 & 0.0 & 0.0 & 0.0 & 0.0 & 0.01 & 0.04 & 0.46 \\
\hline 12.0 & 0.0 & 0.0 & 0.0 & 0.0 & 0.0 & 0.0 & 0.0 & 0.0 & 0.0 & 0.0 & 0.0 & 0.00 & 0.03 & 0.42 \\
\hline 12.5 & 0.0 & 0.0 & 0.0 & 0.0 & 0.0 & 0.0 & 0.0 & 0.0 & 0.0 & 0.0 & 0.0 & 0.00 & 0.02 & 0.39 \\
\hline 13.0 & 0.0 & 0.0 & 0.0 & 0.0 & 0.0 & 0.0 & 0.0 & 0.0 & 0.0 & 0.0 & 0.0 & 0.00 & 0.01 & 0.35 \\
\hline 13.5 & 0.0 & 0.0 & 0.0 & 0.0 & 0.0 & 0.0 & 0.0 & 0.0 & 0.0 & 0.0 & 0.0 & 0.00 & 0.01 & 0.34 \\
\hline 14.0 & 0.0 & 0.0 & 0.0 & 0.0 & 0.0 & 0.0 & 0.0 & 0.0 & 0.0 & 0.0 & 0.0 & 0.00 & 0.01 & 0.29 \\
\hline 14.5 & 0.0 & 0.0 & 0.0 & 0.0 & 0.0 & 0.0 & 0.0 & 0.0 & 0.0 & 0.0 & 0.0 & 0.00 & 0.00 & 0.28 \\
\hline 15.0 & 0.0 & 0.0 & 0.0 & 0.0 & 0.0 & 0.0 & 0.0 & 0.0 & 0.0 & 0.0 & 0.0 & 0.00 & 0.00 & 0.23 \\
\hline 15.5 & 0.0 & 0.0 & 0.0 & 0.0 & 0.0 & 0.0 & 0.0 & 0.0 & 0.0 & 0.0 & 0.0 & 0.0 & 0.00 & 0.22 \\
\hline 16.0 & 0.0 & 0.0 & 0.0 & 0.0 & 0.0 & 0.0 & 0.0 & 0.0 & 0.0 & 0.0 & 0.0 & 0.0 & 0.00 & 0.22 \\
\hline 16.5 & 0.0 & 0.0 & 0.0 & 0.0 & 0.0 & 0.0 & 0.0 & 0.0 & 0.0 & 0.0 & 0.0 & 0.0 & 0.00 & 0.17 \\
\hline 17.0 & 0.0 & 0.0 & 0.0 & 0.0 & 0.0 & 0.0 & 0.0 & 0.0 & 0.0 & 0.0 & 0.0 & 0.0 & 0.00 & 0.17 \\
\hline 17.5 & 0.0 & 0.0 & 0.0 & 0.0 & 0.0 & 0.0 & 0.0 & 0.0 & 0.0 & 0.0 & 0.0 & 0.0 & 0.0 & 0.13 \\
\hline 18.0 & 0.0 & 0.0 & 0.0 & 0.0 & 0.0 & 0.0 & 0.0 & 0.0 & 0.0 & 0.0 & 0.0 & 0.0 & 0.0 & 0.12 \\
\hline 18.5 & 0.0 & 0.0 & 0.0 & 0.0 & 0.0 & 0.0 & 0.0 & 0.0 & 0.0 & 0.0 & 0.0 & 0.0 & 0.0 & 0.09 \\
\hline 19.0 & 0.0 & 0.0 & 0.0 & 0.0 & 0.0 & 0.0 & 0.0 & 0.0 & 0.0 & 0.0 & 0.0 & 0.0 & 0.0 & 0.08 \\
\hline 19.5 & 0.0 & 0.0 & 0.0 & 0.0 & 0.0 & 0.0 & 0.0 & 0.0 & 0.0 & 0.0 & 0.0 & 0.0 & 0.0 & 0.06 \\
\hline 20.0 & 0.0 & 0.0 & 0.0 & 0.0 & 0.0 & 0.0 & 0.0 & 0.0 & 0.0 & 0.0 & 0.0 & 0.0 & 0.0 & 0.05 \\
\hline
\end{tabular}

Notes. The relative angular momentum loss considering particles bound tighter to the central star than to the encounter for all simulated configurations of prograde parabolic $(e=1)$ star-disc encounters. The first row contains the perturber masses $M_{2}$ in units of $M_{1}$, the first column contains the relative periastra $r_{\text {peri }} / r_{\text {disc }}$. 
M. Steinhausen et al.: Disc-mass distribution in star-disc encounters

Table B.3. Relative angular momentum loss assuming an initial disc-mass distribution of $p=1$.

\begin{tabular}{|c|c|c|c|c|c|c|c|c|c|c|c|c|c|c|}
\hline & $0.1 M_{1}$ & $0.3 M_{1}$ & $0.5 M_{1}$ & $1 M_{1}$ & $1.5 M_{1}$ & $2 M_{1}$ & $3 M_{1}$ & $4 M_{1}$ & $5 M_{1}$ & $9 M_{1}$ & $20 M_{1}$ & $50 M_{1}$ & $90 M_{1}$ & $500 M_{1}$ \\
\hline 00.1 & 0.24 & 0.49 & 0.63 & 0.69 & 0.70 & 0.72 & 0.74 & 0.71 & 0.79 & 0.88 & 0.92 & 0.93 & 0.90 & 0.95 \\
\hline 00.2 & 0.21 & 0.40 & 0.59 & 0.71 & 0.82 & 0.86 & 0.86 & 0.87 & 0.90 & 0.94 & 0.95 & 0.94 & 0.94 & 0.97 \\
\hline 00.3 & 0.19 & 0.32 & 0.60 & 0.79 & 0.85 & 0.88 & 0.91 & 0.93 & 0.94 & 0.96 & 0.97 & 0.99 & 0.99 & 0.99 \\
\hline 00.4 & 0.17 & 0.24 & 0.58 & 0.78 & 0.86 & 0.89 & 0.94 & 0.96 & 0.96 & 0.98 & 0.99 & 0.99 & 0.99 & 1.00 \\
\hline 00.5 & 0.16 & 0.19 & 0.55 & 0.78 & 0.86 & 0.89 & 0.92 & 0.94 & 0.95 & 0.97 & 0.99 & 1.00 & 0.99 & 1.00 \\
\hline 00.6 & 0.15 & 0.17 & 0.54 & 0.77 & 0.83 & 0.85 & 0.89 & 0.91 & 0.93 & 0.96 & 0.99 & 0.99 & 1.00 & 1.00 \\
\hline 00.7 & 0.14 & 0.17 & 0.54 & 0.73 & 0.79 & 0.82 & 0.86 & 0.89 & 0.90 & 0.94 & 0.97 & 0.99 & 1.00 & 1.00 \\
\hline 00.8 & 0.14 & 0.19 & 0.49 & 0.69 & 0.75 & 0.79 & 0.83 & 0.86 & 0.88 & 0.93 & 0.96 & 0.99 & 0.99 & 1.00 \\
\hline 00.9 & 0.13 & 0.21 & 0.44 & 0.64 & 0.70 & 0.75 & 0.80 & 0.83 & 0.85 & 0.90 & 0.95 & 0.98 & 0.99 & 1.00 \\
\hline 01.0 & 0.13 & 0.22 & 0.39 & 0.59 & 0.67 & 0.71 & 0.77 & 0.80 & 0.82 & 0.88 & 0.94 & 0.97 & 0.99 & 1.00 \\
\hline 01.5 & 0.05 & 0.16 & 0.20 & 0.35 & 0.45 & 0.51 & 0.60 & 0.65 & 0.68 & 0.77 & 0.86 & 0.92 & 0.95 & 0.99 \\
\hline 02.0 & 0.00 & 0.05 & 0.10 & 0.18 & 0.25 & 0.32 & 0.42 & 0.48 & 0.53 & 0.64 & 0.77 & 0.87 & 0.91 & 0.98 \\
\hline 02.5 & 0.00 & 0.01 & 0.02 & 0.08 & 0.14 & 0.19 & 0.27 & 0.33 & 0.38 & 0.52 & 0.67 & 0.80 & 0.86 & 0.95 \\
\hline 03.0 & 0.00 & 0.00 & 0.00 & 0.02 & 0.06 & 0.09 & 0.14 & 0.20 & 0.24 & 0.39 & 0.56 & 0.73 & 0.81 & 0.94 \\
\hline 03.5 & 0.00 & 0.00 & 0.00 & 0.00 & 0.01 & 0.03 & 0.08 & 0.12 & 0.15 & 0.27 & 0.47 & 0.66 & 0.76 & 0.92 \\
\hline 04.0 & 0.00 & 0.00 & 0.00 & 0.00 & 0.00 & 0.01 & 0.02 & 0.05 & 0.08 & 0.19 & 0.37 & 0.58 & 0.70 & 0.89 \\
\hline 04.5 & 0.00 & 0.00 & 0.00 & 0.00 & 0.00 & 0.00 & 0.01 & 0.02 & 0.04 & 0.11 & 0.28 & 0.51 & 0.63 & 0.86 \\
\hline 05.0 & 0.0 & 0.00 & 0.0 & 0.00 & 0.00 & 0.00 & 0.00 & 0.01 & 0.01 & 0.07 & 0.22 & 0.44 & 0.58 & 0.83 \\
\hline 05.5 & 0.0 & 0.0 & 0.0 & 0.0 & 0.00 & 0.00 & 0.00 & 0.00 & 0.00 & 0.03 & 0.14 & 0.36 & 0.50 & 0.81 \\
\hline 06.0 & 0.0 & 0.0 & 0.0 & 0.0 & 0.00 & 0.00 & 0.00 & 0.00 & 0.00 & 0.01 & 0.10 & 0.31 & 0.44 & 0.77 \\
\hline 06.5 & 0.0 & 0.0 & 0.0 & 0.0 & 0.0 & 0.00 & 0.00 & 0.00 & 0.00 & 0.01 & 0.07 & 0.24 & 0.38 & 0.74 \\
\hline 07.0 & 0.0 & 0.0 & 0.0 & 0.0 & 0.0 & 0.00 & 0.00 & 0.00 & 0.00 & 0.00 & 0.04 & 0.20 & 0.32 & 0.70 \\
\hline 07.5 & 0.0 & 0.0 & 0.0 & 0.0 & 0.0 & 0.0 & 0.0 & 0.0 & 0.00 & 0.00 & 0.02 & 0.14 & 0.28 & 0.67 \\
\hline 08.0 & 0.0 & 0.0 & 0.0 & 0.0 & 0.0 & 0.0 & 0.0 & 0.0 & 0.00 & 0.00 & 0.01 & 0.12 & 0.25 & 0.63 \\
\hline 08.5 & 0.0 & 0.0 & 0.0 & 0.0 & 0.0 & 0.0 & 0.0 & 0.0 & 0.0 & 0.00 & 0.01 & 0.07 & 0.20 & 0.60 \\
\hline 09.0 & 0.0 & 0.0 & 0.0 & 0.0 & 0.0 & 0.0 & 0.0 & 0.0 & 0.0 & 0.00 & 0.00 & 0.05 & 0.17 & 0.56 \\
\hline 09.5 & 0.0 & 0.0 & 0.0 & 0.0 & 0.0 & 0.0 & 0.0 & 0.0 & 0.0 & 0.0 & 0.00 & 0.04 & 0.12 & 0.53 \\
\hline 10.0 & 0.0 & 0.0 & 0.0 & 0.0 & 0.0 & 0.0 & 0.0 & 0.0 & 0.0 & 0.0 & 0.00 & 0.02 & 0.11 & 0.49 \\
\hline 10.5 & 0.0 & 0.0 & 0.0 & 0.0 & 0.0 & 0.0 & 0.0 & 0.0 & 0.0 & 0.0 & 0.0 & 0.02 & 0.07 & 0.45 \\
\hline 11.0 & 0.0 & 0.0 & 0.0 & 0.0 & 0.0 & 0.0 & 0.0 & 0.0 & 0.0 & 0.0 & 0.0 & 0.01 & 0.06 & 0.42 \\
\hline 11.5 & 0.0 & 0.0 & 0.0 & 0.0 & 0.0 & 0.0 & 0.0 & 0.0 & 0.0 & 0.0 & 0.0 & 0.01 & 0.03 & 0.38 \\
\hline 12.0 & 0.0 & 0.0 & 0.0 & 0.0 & 0.0 & 0.0 & 0.0 & 0.0 & 0.0 & 0.0 & 0.0 & 0.00 & 0.03 & 0.35 \\
\hline 12.5 & 0.0 & 0.0 & 0.0 & 0.0 & 0.0 & 0.0 & 0.0 & 0.0 & 0.0 & 0.0 & 0.0 & 0.00 & 0.01 & 0.32 \\
\hline 13.0 & 0.0 & 0.0 & 0.0 & 0.0 & 0.0 & 0.0 & 0.0 & 0.0 & 0.0 & 0.0 & 0.0 & 0.00 & 0.01 & 0.29 \\
\hline 13.5 & 0.0 & 0.0 & 0.0 & 0.0 & 0.0 & 0.0 & 0.0 & 0.0 & 0.0 & 0.0 & 0.0 & 0.00 & 0.01 & 0.28 \\
\hline 14.0 & 0.0 & 0.0 & 0.0 & 0.0 & 0.0 & 0.0 & 0.0 & 0.0 & 0.0 & 0.0 & 0.0 & 0.00 & 0.00 & 0.24 \\
\hline 14.5 & 0.0 & 0.0 & 0.0 & 0.0 & 0.0 & 0.0 & 0.0 & 0.0 & 0.0 & 0.0 & 0.0 & 0.00 & 0.00 & 0.23 \\
\hline 15.0 & 0.0 & 0.0 & 0.0 & 0.0 & 0.0 & 0.0 & 0.0 & 0.0 & 0.0 & 0.0 & 0.0 & 0.00 & 0.00 & 0.19 \\
\hline 15.5 & 0.0 & 0.0 & 0.0 & 0.0 & 0.0 & 0.0 & 0.0 & 0.0 & 0.0 & 0.0 & 0.0 & 0.0 & 0.00 & 0.18 \\
\hline 16.0 & 0.0 & 0.0 & 0.0 & 0.0 & 0.0 & 0.0 & 0.0 & 0.0 & 0.0 & 0.0 & 0.0 & 0.0 & 0.00 & 0.17 \\
\hline 16.5 & 0.0 & 0.0 & 0.0 & 0.0 & 0.0 & 0.0 & 0.0 & 0.0 & 0.0 & 0.0 & 0.0 & 0.0 & 0.00 & 0.14 \\
\hline 17.0 & 0.0 & 0.0 & 0.0 & 0.0 & 0.0 & 0.0 & 0.0 & 0.0 & 0.0 & 0.0 & 0.0 & 0.0 & 0.00 & 0.13 \\
\hline 17.5 & 0.0 & 0.0 & 0.0 & 0.0 & 0.0 & 0.0 & 0.0 & 0.0 & 0.0 & 0.0 & 0.0 & 0.0 & 0.0 & 0.10 \\
\hline 18.0 & 0.0 & 0.0 & 0.0 & 0.0 & 0.0 & 0.0 & 0.0 & 0.0 & 0.0 & 0.0 & 0.0 & 0.0 & 0.0 & 0.10 \\
\hline 18.5 & 0.0 & 0.0 & 0.0 & 0.0 & 0.0 & 0.0 & 0.0 & 0.0 & 0.0 & 0.0 & 0.0 & 0.0 & 0.0 & 0.07 \\
\hline 19.0 & 0.0 & 0.0 & 0.0 & 0.0 & 0.0 & 0.0 & 0.0 & 0.0 & 0.0 & 0.0 & 0.0 & 0.0 & 0.0 & 0.07 \\
\hline 19.5 & 0.0 & 0.0 & 0.0 & 0.0 & 0.0 & 0.0 & 0.0 & 0.0 & 0.0 & 0.0 & 0.0 & 0.0 & 0.0 & 0.05 \\
\hline 20.0 & 0.0 & 0.0 & 0.0 & 0.0 & 0.0 & 0.0 & 0.0 & 0.0 & 0.0 & 0.0 & 0.0 & 0.0 & 0.0 & 0.04 \\
\hline
\end{tabular}

Notes. The relative angular momentum loss considering particles bound tighter to the central star than to the encounter for all simulated configurations of prograde parabolic $(e=1)$ star-disc encounters. The first row contains the perturber masses $M_{2}$ in units of $M_{1}$, the first column contains the relative periastra $r_{\text {peri }} / r_{\text {disc }}$. 
Table B.4. Relative angular momentum loss assuming an initial disc-mass distribution of $p=7 / 4$.

\begin{tabular}{|c|c|c|c|c|c|c|c|c|c|c|c|c|c|c|}
\hline & $0.1 M_{1}$ & $0.3 M_{1}$ & $0.5 M_{1}$ & $1 M_{1}$ & $1.5 M_{1}$ & $2 M_{1}$ & $3 M_{1}$ & $4 M_{1}$ & $5 M_{1}$ & $9 M_{1}$ & $20 M_{1}$ & $50 M_{1}$ & $90 M_{1}$ & $500 M_{1}$ \\
\hline 00.1 & 0.23 & 0.44 & 0.62 & 0.70 & 0.75 & 0.76 & 0.78 & 0.77 & 0.83 & 0.90 & 0.93 & 0.94 & 0.92 & 0.95 \\
\hline 00.2 & 0.20 & 0.35 & 0.59 & 0.75 & 0.84 & 0.88 & 0.89 & 0.90 & 0.92 & 0.95 & 0.96 & 0.96 & 0.96 & 0.98 \\
\hline 00.3 & 0.17 & 0.28 & 0.57 & 0.77 & 0.84 & 0.88 & 0.91 & 0.93 & 0.95 & 0.97 & 0.98 & 0.99 & 0.99 & 0.99 \\
\hline 00.4 & 0.15 & 0.22 & 0.53 & 0.73 & 0.81 & 0.85 & 0.90 & 0.93 & 0.93 & 0.96 & 0.99 & 0.99 & 1.00 & 1.00 \\
\hline 00.5 & 0.14 & 0.18 & 0.48 & 0.69 & 0.77 & 0.81 & 0.85 & 0.88 & 0.91 & 0.94 & 0.98 & 1.00 & 0.99 & 1.00 \\
\hline 00.6 & 0.13 & 0.16 & 0.45 & 0.65 & 0.72 & 0.75 & 0.81 & 0.83 & 0.86 & 0.92 & 0.97 & 0.99 & 0.99 & 1.00 \\
\hline 00.7 & 0.12 & 0.16 & 0.43 & 0.60 & 0.67 & 0.70 & 0.75 & 0.79 & 0.82 & 0.87 & 0.94 & 0.99 & 0.99 & 1.00 \\
\hline 00.8 & 0.11 & 0.17 & 0.38 & 0.55 & 0.62 & 0.66 & 0.72 & 0.75 & 0.77 & 0.85 & 0.92 & 0.97 & 0.99 & 1.00 \\
\hline 00.9 & 0.10 & 0.17 & 0.34 & 0.50 & 0.57 & 0.62 & 0.67 & 0.71 & 0.74 & 0.81 & 0.89 & 0.96 & 0.98 & 1.00 \\
\hline 01.0 & 0.09 & 0.17 & 0.29 & 0.45 & 0.52 & 0.57 & 0.63 & 0.67 & 0.70 & 0.78 & 0.86 & 0.93 & 0.97 & 1.00 \\
\hline 01.5 & 0.03 & 0.10 & 0.14 & 0.25 & 0.32 & 0.37 & 0.45 & 0.50 & 0.53 & 0.63 & 0.74 & 0.83 & 0.89 & 0.99 \\
\hline 02.0 & 0.00 & 0.03 & 0.06 & 0.12 & 0.17 & 0.22 & 0.29 & 0.34 & 0.38 & 0.49 & 0.62 & 0.74 & 0.81 & 0.95 \\
\hline 02.5 & 0.00 & 0.00 & 0.01 & 0.05 & 0.09 & 0.12 & 0.18 & 0.22 & 0.26 & 0.37 & 0.51 & 0.65 & 0.74 & 0.90 \\
\hline 03.0 & 0.00 & 0.00 & 0.00 & 0.01 & 0.04 & 0.06 & 0.09 & 0.13 & 0.16 & 0.27 & 0.41 & 0.58 & 0.67 & 0.86 \\
\hline 03.5 & 0.00 & 0.00 & 0.00 & 0.00 & 0.01 & 0.02 & 0.05 & 0.07 & 0.10 & 0.18 & 0.33 & 0.50 & 0.60 & 0.82 \\
\hline 04.0 & 0.00 & 0.00 & 0.00 & 0.00 & 0.00 & 0.00 & 0.01 & 0.03 & 0.05 & 0.13 & 0.25 & 0.43 & 0.54 & 0.77 \\
\hline 04.5 & 0.00 & 0.00 & 0.00 & 0.00 & 0.00 & 0.00 & 0.00 & 0.01 & 0.02 & 0.07 & 0.19 & 0.36 & 0.47 & 0.73 \\
\hline 05.0 & 0.0 & 0.00 & 0.0 & 0.00 & 0.00 & 0.00 & 0.00 & 0.00 & 0.01 & 0.04 & 0.14 & 0.30 & 0.42 & 0.69 \\
\hline 05.5 & 0.0 & 0.0 & 0.0 & 0.0 & 0.00 & 0.00 & 0.00 & 0.00 & 0.00 & 0.02 & 0.09 & 0.25 & 0.36 & 0.66 \\
\hline 06.0 & 0.0 & 0.0 & 0.0 & 0.0 & 0.00 & 0.00 & 0.00 & 0.00 & 0.00 & 0.01 & 0.07 & 0.20 & 0.31 & 0.62 \\
\hline 06.5 & 0.0 & 0.0 & 0.0 & 0.0 & 0.0 & 0.00 & 0.00 & 0.00 & 0.00 & 0.00 & 0.05 & 0.15 & 0.26 & 0.58 \\
\hline 07.0 & 0.0 & 0.0 & 0.0 & 0.0 & 0.0 & 0.00 & 0.00 & 0.00 & 0.00 & 0.00 & 0.02 & 0.13 & 0.22 & 0.54 \\
\hline 07.5 & 0.0 & 0.0 & 0.0 & 0.0 & 0.0 & 0.0 & 0.0 & 0.0 & 0.00 & 0.00 & 0.01 & 0.09 & 0.18 & 0.51 \\
\hline 08.0 & 0.0 & 0.0 & 0.0 & 0.0 & 0.0 & 0.0 & 0.0 & 0.0 & 0.00 & 0.00 & 0.01 & 0.07 & 0.16 & 0.47 \\
\hline 08.5 & 0.0 & 0.0 & 0.0 & 0.0 & 0.0 & 0.0 & 0.0 & 0.0 & 0.0 & 0.00 & 0.00 & 0.04 & 0.13 & 0.44 \\
\hline 09.0 & 0.0 & 0.0 & 0.0 & 0.0 & 0.0 & 0.0 & 0.0 & 0.0 & 0.0 & 0.00 & 0.00 & 0.03 & 0.11 & 0.41 \\
\hline 09.5 & 0.0 & 0.0 & 0.0 & 0.0 & 0.0 & 0.0 & 0.0 & 0.0 & 0.0 & 0.0 & 0.00 & 0.03 & 0.08 & 0.38 \\
\hline 10.0 & 0.0 & 0.0 & 0.0 & 0.0 & 0.0 & 0.0 & 0.0 & 0.0 & 0.0 & 0.0 & 0.00 & 0.01 & 0.07 & 0.34 \\
\hline 10.5 & 0.0 & 0.0 & 0.0 & 0.0 & 0.0 & 0.0 & 0.0 & 0.0 & 0.0 & 0.0 & 0.0 & 0.01 & 0.04 & 0.31 \\
\hline 11.0 & 0.0 & 0.0 & 0.0 & 0.0 & 0.0 & 0.0 & 0.0 & 0.0 & 0.0 & 0.0 & 0.0 & 0.00 & 0.04 & 0.29 \\
\hline 11.5 & 0.0 & 0.0 & 0.0 & 0.0 & 0.0 & 0.0 & 0.0 & 0.0 & 0.0 & 0.0 & 0.0 & 0.00 & 0.02 & 0.26 \\
\hline 12.0 & 0.0 & 0.0 & 0.0 & 0.0 & 0.0 & 0.0 & 0.0 & 0.0 & 0.0 & 0.0 & 0.0 & 0.00 & 0.02 & 0.23 \\
\hline 12.5 & 0.0 & 0.0 & 0.0 & 0.0 & 0.0 & 0.0 & 0.0 & 0.0 & 0.0 & 0.0 & 0.0 & 0.00 & 0.01 & 0.22 \\
\hline 13.0 & 0.0 & 0.0 & 0.0 & 0.0 & 0.0 & 0.0 & 0.0 & 0.0 & 0.0 & 0.0 & 0.0 & 0.00 & 0.01 & 0.19 \\
\hline 13.5 & 0.0 & 0.0 & 0.0 & 0.0 & 0.0 & 0.0 & 0.0 & 0.0 & 0.0 & 0.0 & 0.0 & 0.00 & 0.01 & 0.18 \\
\hline 14.0 & 0.0 & 0.0 & 0.0 & 0.0 & 0.0 & 0.0 & 0.0 & 0.0 & 0.0 & 0.0 & 0.0 & 0.00 & 0.00 & 0.15 \\
\hline 14.5 & 0.0 & 0.0 & 0.0 & 0.0 & 0.0 & 0.0 & 0.0 & 0.0 & 0.0 & 0.0 & 0.0 & 0.00 & 0.00 & 0.15 \\
\hline 15.0 & 0.0 & 0.0 & 0.0 & 0.0 & 0.0 & 0.0 & 0.0 & 0.0 & 0.0 & 0.0 & 0.0 & 0.00 & 0.00 & 0.12 \\
\hline 15.5 & 0.0 & 0.0 & 0.0 & 0.0 & 0.0 & 0.0 & 0.0 & 0.0 & 0.0 & 0.0 & 0.0 & 0.0 & 0.00 & 0.12 \\
\hline 16.0 & 0.0 & 0.0 & 0.0 & 0.0 & 0.0 & 0.0 & 0.0 & 0.0 & 0.0 & 0.0 & 0.0 & 0.0 & 0.00 & 0.11 \\
\hline 16.5 & 0.0 & 0.0 & 0.0 & 0.0 & 0.0 & 0.0 & 0.0 & 0.0 & 0.0 & 0.0 & 0.0 & 0.0 & 0.00 & 0.09 \\
\hline 17.0 & 0.0 & 0.0 & 0.0 & 0.0 & 0.0 & 0.0 & 0.0 & 0.0 & 0.0 & 0.0 & 0.0 & 0.0 & 0.00 & 0.09 \\
\hline 17.5 & 0.0 & 0.0 & 0.0 & 0.0 & 0.0 & 0.0 & 0.0 & 0.0 & 0.0 & 0.0 & 0.0 & 0.0 & 0.0 & 0.06 \\
\hline 18.0 & 0.0 & 0.0 & 0.0 & 0.0 & 0.0 & 0.0 & 0.0 & 0.0 & 0.0 & 0.0 & 0.0 & 0.0 & 0.0 & 0.06 \\
\hline 18.5 & 0.0 & 0.0 & 0.0 & 0.0 & 0.0 & 0.0 & 0.0 & 0.0 & 0.0 & 0.0 & 0.0 & 0.0 & 0.0 & 0.04 \\
\hline 19.0 & 0.0 & 0.0 & 0.0 & 0.0 & 0.0 & 0.0 & 0.0 & 0.0 & 0.0 & 0.0 & 0.0 & 0.0 & 0.0 & 0.04 \\
\hline 19.5 & 0.0 & 0.0 & 0.0 & 0.0 & 0.0 & 0.0 & 0.0 & 0.0 & 0.0 & 0.0 & 0.0 & 0.0 & 0.0 & 0.03 \\
\hline 20.0 & 0.0 & 0.0 & 0.0 & 0.0 & 0.0 & 0.0 & 0.0 & 0.0 & 0.0 & 0.0 & 0.0 & 0.0 & 0.0 & 0.03 \\
\hline
\end{tabular}

Notes. The relative angular momentum loss considering particles bound tighter to the central star than to the encounter for all simulated configurations of prograde parabolic $(e=1)$ star-disc encounters. The first row contains the perturber masses $M_{2}$ in units of $M_{1}$, the first column contains the relative periastra $r_{\text {peri }} / r_{\text {disc }}$. 